\title{
Approximating fixed points of amenable semigroup and infinite family of nonexpansive mappings and solving systems of variational inequalities and systems of equilibrium problems
}

Hossein Piri

Correspondence:

hossein_piri1979@yahoo.com

Department of Mathematics, University of Bonab 55517-61167 Bonab, Iran

\begin{abstract}
We introduce an iterative scheme for finding a common element of the set of solutions for systems of equilibrium problems and systems of variational inequalities and the set of common fixed points for an infinite family and left amenable semigroup of nonexpansive mappings in Hilbert spaces. The results presented in this paper mainly extend and improved some well-known results in the literature.

Mathematics Subject Classification (2000): 47H09; 47H10; 47H20; 43A07; 47J25.

Keywords: common fixed point, strong convergence, amenable semigroup, explicit iterative, system of equilibrium problem.
\end{abstract}

\section{Introduction}

Let $H$ be a real Hilbert space and let $C$ be a nonempty closed convex subset of $H$.

Let $A: C \rightarrow H$ be a nonlinear mapping. The classical variational inequality problem is to fined $x \in C$ such that

$$
\langle A x, y-x\rangle \geq 0, \quad \forall y \in C .
$$

The set of solution of $(1)$ is denoted by $\operatorname{VI}(C, A)$, i.e.,

$$
\operatorname{VI}(C, A)=\{x \in C:\langle A x, y-x\rangle \geq 0, \quad \forall y \in C\} .
$$

Recall that the following definitions:

(1) $A$ is called monotone if

$$
\langle A x-A y, x-y\rangle \geq 0, \quad \forall x, y \in C .
$$

(2) $A$ is called $\alpha$-strongly monotone if there exists a positive constant $\alpha$ such that

$$
\langle A x-A y, x-y\rangle \geq \alpha\|x-y\|^{2}, \quad \forall x, y \in C .
$$


(3) $A$ is called $\mu$-Lipschitzian if there exist a positive constant $\mu$ such that

$$
\|A x-A y\| \leq \mu\|x-y\|, \quad \forall x, y \in C .
$$

(4) $A$ is called $\alpha$-inverse strongly monotone, if there exists a positive real number $\alpha>0$

such that

$$
\langle A x-A y, x-y\rangle \geq \alpha\|A x-A y\|^{2}, \quad \forall x, y \in C .
$$

It is obvious that any $\alpha$-inverse strongly monotone mapping $B$ is $\frac{1}{\alpha}$-Lipschitzian.

(5) A mapping $T: C \rightarrow C$ is called nonexpansive if $\|T x-T y\| \leq\|x-y\|$ for all $x, y$

$\in C$. Next, we denote by $\operatorname{Fix}(T)$ the set of fixed point of $T$.

(6) A mapping $f: C \rightarrow C$ is said to be contraction if there exists a coefficient $\alpha \in$

$(0,1)$ such that

$$
\|f(x)-f(y)\| \leq \alpha\|x-y\|, \quad \forall x, y \in C .
$$

(7) A set-valued mapping $U: H \rightarrow 2^{H}$ is called monotone if for all $x, y \in H, f \in U_{x}$ and $g \in U y$ imply $\langle x-y, f-g\rangle \geq 0$.

(8) A monotone mapping $U: H \rightarrow 2^{H}$ is maximal if the graph $G(U)$ of $U$ is not properly contained in the graph of any other monotone mapping.

It is known that a monotone mapping $U$ is maximal if and only if for $(x, f) \in H \times H$, $\langle x-y, f-g\rangle \leq 0$ for every $(y, g) \in G(U)$ implies that $f \in U x$. Let $B$ be a monotone mapping of $C$ into $H$ and let $N_{C} x$ be the normal cone to $C$ at $x \in C$, that is, $N_{C} x=\{y \in H$ : $\langle x-z, y\rangle \leq 0, \forall z \in C\}$ and define

$$
U x= \begin{cases}B x+N_{C} x, & x \in C, \\ \varnothing & x \notin C .\end{cases}
$$

Then $U$ is the maximal monotone and $0 \in U x$ if and only if $x \in \mathrm{VI}(C, B)$; see [1].

Let $F$ be a bi-function of $C \times C$ into $\mathbb{R}$, where $\mathbb{R}$ is the set of real numbers. The equilibrium problem for $F: C \times C \rightarrow \mathbb{R}$ is to determine its equilibrium points, i.e the set

$$
\mathrm{EP}(F)=\{x \in C: F(x, y) \geq 0, \forall y \in C\} .
$$

Let $\mathcal{J}=\left\{F_{i}\right\}_{i \in I}$ be a family of bi-functions from $C \times C$ into $\mathbb{R}$. The system of equilibrium problems for $\mathcal{J}=\left\{F_{i}\right\}_{i \in I}$ is to determine common equilibrium points for $\mathcal{J}=\left\{F_{i}\right\}_{i \in I}$, i.e the set

$$
\mathrm{EP}(\mathcal{J})=\left\{x \in C: F_{i}(x, y) \geq 0, \forall y \in C, \forall i \in I\right\} .
$$

Numerous problems in physics, optimization, and economics reduce into finding some element of $\operatorname{EP}(F)$. Some method have been proposed to solve the equilibrium problem; see, for instance [2-5]. The formulation (3), extend this formalism to systems of such problems, covering in particular various forms of feasibility problems [6,7]. 
Given any $r>0$ the operator $J_{r}^{F}: H \rightarrow C$ defined by

$$
J_{r}^{F}(x)=\left\{z \in C: F(z, y)+\frac{1}{r}\langle y-z, z-x\rangle \geq 0, \forall y \in C\right\},
$$

is called the resolvent of $F$, see [3]. It is shown [3] that under suitable hypotheses on $F$ (to be stated precisely in Sect. 2), $J_{r}^{F}: H \rightarrow C$ is single- valued and firmly nonexpansive and

satisfies

$$
\operatorname{Fix}\left(J_{r}^{F}\right)=\operatorname{EP}(F), \quad \forall r>0 .
$$

Using this result, in 2007, Yao et al. [8], proposed the following explicit scheme with respect to $W$-mappings for an infinite family of nonexpansive mappings:

$$
x_{n+1}=\alpha_{n} f\left(x_{n}\right)+\beta_{n} x_{n}+\gamma_{n} W_{n} J_{r_{n}}^{F} x_{n}
$$

They proved that if the sequences $\left\{\alpha_{n}\right\},\left\{\beta_{n}\right\},\left\{\gamma_{n}\right\}$ and $\left\{r_{n}\right\}$ of parameters satisfy appropriate conditions, then, the sequences $\left\{x_{n}\right\}$ and $\left\{J_{r_{n}}^{F} x_{n}\right\}$ both converge strongly to the unique $x^{*} \in \cap_{i=1}^{\infty} \operatorname{Fix}\left(T_{i}\right) \cap \operatorname{EP}(F)$, where $x^{*} \in P_{\bigcap_{i=1}^{\infty} \operatorname{Fix}\left(T_{i}\right) \cap \operatorname{EP}_{(F)}} f\left(x^{*}\right)$. Their results extend and improve the corresponding results announced by Combettes and Hirstoaga [3] and Takahashi and Takahashi [5].

Very recently, Jitpeera et al. [9], introduced the iterative scheme based on viscosity and Cesàro mean

$$
\left\{\begin{array}{l}
\phi\left(u_{n}, y\right)+\varphi(y)-\varphi\left(u_{n}\right)+\frac{1}{r_{n}}\left\langle y-u_{n}, u_{n}-x_{n}\right\rangle \geq 0, \quad \forall y \in C, \\
y_{n}=\delta_{n} u_{n}+\left(1-\delta_{n}\right) P_{C}\left(u_{n}-\lambda_{n} B u_{n}\right) \\
x_{n+1}=\alpha_{n} \gamma f\left(x_{n}\right)+\beta_{n} x_{n}+\left(\left(1-\beta_{n}\right) I-\alpha_{n} A\right) \frac{1}{n+1} \sum_{i=0}^{n} T^{i} y_{n}, \quad \forall n \geq 0
\end{array}\right.
$$

where $B: C \rightarrow H$ is $\beta$-inverse strongly monotone, $\phi: C \rightarrow \mathbb{R} \cup\{\infty\}$ is a proper lower semi-continuous and convex function, $T^{i}: C \rightarrow C$ is a nonexpansive mapping for all $i$ $=1,2, \ldots, n,\left\{\alpha_{n}\right\},\left\{\beta_{n}\right\},\left\{\delta_{n}\right\} \subset(0,1),\left\{\lambda_{n}\right\} \subset(0,2 \beta)$ and $\left\{r_{n}\right\} \subset(0, \infty)$ satisfy the following conditions

(i) $\lim _{n \rightarrow \infty} \alpha_{n}=0, \sum_{n=1}^{\infty} \alpha_{n}=\infty$,

(ii) $\lim _{n \rightarrow \infty} \delta_{n}=0$

(iii) $0<\lim \inf _{n \rightarrow \infty} \beta_{n} \leq \lim \sup _{n \rightarrow \infty} \beta_{n}<1$.

(iv) $\left\{\lambda_{n}\right\} \subset[a, b] \subset(0,2 \beta)$ and $\lim _{\inf _{n \rightarrow \infty}}\left|\lambda_{n+1}-\lambda_{n}\right|=0$,

(v) $\lim \inf _{n \rightarrow \infty} r_{n}>0$ and $\lim _{\inf _{n \rightarrow \infty}}\left|r_{n+1}-r_{n}\right|=0$.

They show that if $\theta=\bigcap_{i=1}^{n} \operatorname{Fix}\left(T^{i}\right) \cap \operatorname{VI}(C, B) \cap \operatorname{MEP}(\phi, \varphi)$ is nonempty, then the sequence $\left\{x_{n}\right\}$ converges strongly to the $z=P_{\theta}(I-A+\gamma f) z$ which is the unique solution of the variational inequality

$$
\langle(\gamma f-A) z, x-z\rangle \leq 0 \quad \forall y \in \theta .
$$

In this paper, motivated and inspired by Yao et al. [8,10-15], Lau et al. [16], Jitpeera et al. [9], Kangtunyakarn [17] and Kim [18], Atsushiba and Takahashi [19], Saeidi [20], Piri [21-23] and Piri and Badali [24], we introduce the following iterative scheme for finding a common element of the set of solutions for a system of equilibrium problems 
$\mathcal{J}=\left\{F_{k}: k=1,2,3, \ldots, M\right\}$ for a family $\mathcal{J}=\left\{F_{k}: k=1,2,3, \ldots, M\right\}$ of equilibrium bifunctions, systems of variational inequalities, the set of common fixed points for an infinite family $\psi=\left\{T_{i}, i=1,2, \ldots\right\}$ of nonexpansive mappings and a left amenable semigroup $\phi=\left\{T_{t}: t \in S\right\}$ of nonexpansive mappings, with respect to $W$-mappings and a left regular sequence $\left\{\mu_{n}\right\}$ of means defined on an appropriate space of bounded real-valued functions of the semigroup

$$
\left\{\begin{array}{l}
z_{n}=J_{r_{M}, n}^{F_{M}} \ldots J_{r_{2}, n}^{F_{2}} J_{r_{1}, n}^{F_{1}} x_{n}, \\
y_{n}=\eta_{n} P_{C}\left(z_{n}-\zeta_{n} A z_{n}\right)+\left(1-\eta_{n}\right) P_{C}\left(z_{n}-\delta_{n} B z_{n}\right), \\
x_{n+1}=\alpha_{n} f\left(T_{\mu_{n}} W_{n} y_{n}\right)+\beta_{n} x_{n}+\gamma_{n} T_{\mu_{n}} W_{n} y_{n}, \quad n \geq 1,
\end{array}\right.
$$

where $A: C \rightarrow H$ be $\beta$-inverse monotone map and $B: C \rightarrow H$ be $\delta$-inverse monotone map. We prove that under mild assumptions on parameters like that in Yao et al. [8],

the sequences $\left\{x_{n}\right\}$ and $\left\{J_{r_{k, n}}^{F_{k}} x_{n}\right\}_{k=1}^{M}$ converge strongly to $x^{*} \in \mathcal{F}=\cap_{i=1}^{\infty} \operatorname{Fix}\left(T_{i}\right) \cap \operatorname{Fix}(\varphi) \cap \operatorname{EP}(\mathcal{J}) \cap \operatorname{VI}(C, A) \cap \operatorname{VI}(C, B)$, where $x^{*}=P_{\mathcal{F}} f\left(x^{*}\right)$.

Compared to the similar works, our results have the merit of studying the solutions of systems of equilibrium problems, systems of variational inequalities and fixed point problems of amenable semigroup of nonexpansive mappings. Consequence for nonnegative integer numbers is also presented.

\section{Preliminaries}

Let $S$ be a semigroup and let $B(S)$ be the space of all bounded real valued functions defined on $S$ with supremum norm. For $s \in S$ and $f \in B(S)$, we define elements $l_{s} f$ and $r_{s} f$ in $B(S)$ by

$$
\left(l_{s} f\right)(t)=f(s t), \quad\left(r_{s} f\right)(t)=f(t s), \quad \forall t \in S .
$$

Let $X$ be a subspace of $B(S)$ containing 1 and let $X^{*}$ be its topological dual. An element $\mu$ of $X^{*}$ is said to be a mean on $X$ if $\|\mu\|=\mu(1)=1$. We often write $\mu_{t}(f(t))$ instead of $\mu(f)$ for $\mu \in X^{*}$ and $f \in X$. Let $X$ be left invariant (respectively right invariant), i.e., $l_{s}(X) \subset X$ (respectively $r_{s}(X) \subset X$ ) for each $s \in S$. A mean $\mu$ on $X$ is said to be left invariant (respectively right invariant) if $\mu\left(l_{s} f\right)=\mu(f)$ (respectively $\mu\left(r_{s} f\right)=\mu(f)$ ) for each $s \in S$ and $f \in X . X$ is said to be left (respectively right) amenable if $X$ has a left (respectively right) invariant mean. $X$ is amenable if $X$ is both left and right amenable. As is well known, $B(S)$ is amenable when $S$ is a commutative semigroup, see [25]. A net $\left\{\mu_{\alpha}\right\}$ of means on $X$ is said to be strongly left regular if

$$
\lim _{\alpha}\left\|l_{s}^{*} \mu_{\alpha}-\mu_{\alpha}\right\|=0
$$

for each $s \in S$, where $l_{s}^{*}$ is the adjoint operator of $l_{s}$.

Let $S$ be a semigroup and let $C$ be a nonempty closed and convex subset of a reflexive Banach space $E$. A family $\phi=\left\{T_{t}: t \in S\right\}$ of mapping from $C$ into itself is said to be a nonexpansive semigroup on $C$ if $T_{t}$ is nonexpansive and $T_{t s}=T_{t} T_{s}$ for each $t, s \in$ $S$. By $\operatorname{Fix}(\phi)$ we denote the set of common fixed points of $\phi$, i.e.

$$
\operatorname{Fix}(\varphi)=\bigcap_{t \in S}\left\{x \in C: T_{t} x=x\right\} .
$$


Lemma 2.1. [25] Let $S$ be a semigroup and $C$ be a nonempty closed convex subset of a reflexive Banach space E. Let $\phi=\left\{T_{t}: t \in S\right\}$ be a nonexpansive semigroup on $H$ such that $\left\{T_{t} x: t \in S\right\}$ is bounded for some $x \in C$, let $X$ be a subspace of $B(S)$ such that $1 \in$ $X$ and the mapping $t \rightarrow\left\langle T_{t} x, y^{*}\right\rangle$ is an element of $X$ for each $x \in C$ and $y^{*} \in E^{*}$, and $\mu$ is a mean on $X$. If we write $T_{\mu} x$ instead of $\int T_{t} x d \mu(t)$, then the followings hold.

(i) $T_{\mu}$ is nonexpansive mapping from $C$ into $C$.

(ii) $T_{\mu} x=x$ for each $x \in \operatorname{Fix}(\phi)$.

(iii) $T_{\mu} x \in \overline{c o}\left\{T_{t} x: t \in S\right\}$ for each $x \in C$.

Let $C$ be a nonempty subset of a Hilbert space $H$ and $T: C \rightarrow H$ a mapping. Then $T$ is said to be demiclosed at $v \in H$ if, for any sequence $\left\{x_{n}\right\}$ in $C$, the following implication holds:

$$
x_{n} \rightarrow u \in C, \quad T x_{n} \rightarrow v \quad \text { imply } \quad T u=v,
$$

where $\rightarrow$ (respectively $\rightarrow$ ) denotes strong (respectively weak) convergence.

Lemma 2.2. [26] Let $C$ be a nonempty closed convex subset of a Hilbert space $H$ and suppose that $T: C \rightarrow H$ is nonexpansive. then, the mapping $I-T$ is demiclosed at zero.

Lemma 2.3. [27] For a given $x \in H, y \in C$,

$$
y=P_{C} x \Leftrightarrow\langle y-x, z-y\rangle \geq 0, \quad \forall z \in C .
$$

It is well known that $P_{C}$ is a firmly nonexpansive mapping of $H$ onto $C$ and satisfies

$$
\left\|P_{C} x-P_{C} y\right\|^{2} \leq\left\langle P_{C} x-P_{C} y, x-y\right\rangle, \quad \forall x, y \in H .
$$

Moreover, $P_{C}$ is characterized by the following properties: $P_{C} x \in C$ and for all $x \in H$, $y \in C$,

$$
\left\langle x-P_{C} x, y-P_{C} x\right\rangle \leq 0 .
$$

It is easy to see that (7) is equivalent to the following inequality

$$
\|x-y\|^{2} \geq\left\|x-P_{C} x\right\|^{2}+\left\|y-P_{C} x\right\|^{2} .
$$

Using Lemma 2.3, one can see that the variational inequality (1) is equivalent to a fixed point problem. It is easy to see that the following is true:

$$
u \in \operatorname{VI}(C, A) \Leftrightarrow u=P_{C}(u-\lambda A u), \quad \lambda>0 .
$$

Lemma 2.4. [28] Let $\left\{x_{n}\right\}$ and $\left\{y_{n}\right\}$ be bounded sequences in a Banach space $E$ and let $\left\{\alpha_{n}\right\}$ be a sequence in $[0,1]$ with $0<\liminf _{n \rightarrow \infty} \alpha_{n} \leq \limsup _{n \rightarrow \infty} \alpha_{n}<1$. Suppose $x_{n+1}=\alpha_{n} x_{n}$ $+\left(1-\alpha_{n}\right) y_{n}$ for all integers $n \geq 0$ and

$$
\limsup _{n \rightarrow \infty}\left(\left\|y_{n+1}-y_{n}\right\|-\left\|x_{n+1}-x_{n}\right\|\right) \leq 1 .
$$

Then, $\lim _{n \rightarrow \infty}\left\|y_{n}-x_{n}\right\|=0$.

Let $F: C \times C \rightarrow \mathbb{R}$ be a bi-function. Given any $r>0$, the operator $J_{r}^{F}: H \rightarrow C$ defined by

$$
J_{r}^{F} x=\left\{z \in C: F(z, y)+\frac{1}{r}\langle y-z, z-x\rangle \geq 0, \forall y \in C\right\}
$$


is called the resolvent of $F$, see [3]. The equilibrium problem for $F$ is to determine its equilibrium points, i.e., the set

$$
\mathrm{EP}(F)=\{x \in C: F(x, y) \geq 0, \forall y \in C\} .
$$

Let $\mathcal{J}=\left\{F_{i}\right\}_{i \in I}$ be a family of bi-functions from $C \times C$ into $\mathbb{R}$. The system of equilibrium problems for $\mathcal{J}$ is to determine common equilibrium points for $\mathcal{J}=\left\{F_{i}\right\}_{i \in I}$. i.e, the set

$$
\mathrm{EP}(\mathcal{J})=\left\{x \in C: F_{i}(x, y) \geq 0, \forall y \in C, \forall i \in I\right\}
$$

Lemma 2.5. [3] Let $C$ be a nonempty closed convex subset of $H$ and $F: C \times C \rightarrow \mathbb{R}$ satisfy

$\left(\mathrm{A}_{1}\right) F(x, x)=0$ for all $x \in C$,

$\left(\mathrm{A}_{2}\right) F$ is monotone, i.e, $F(x, y)+F(y, x) \leq 0$ for all $x, y \in C$,

$\left(\mathrm{A}_{3}\right)$ for all $x, y, z \in C, \lim _{t \rightarrow 0} F(t z+(1-t) x, y) \leq F(x, y)$,

$\left(\mathrm{A}_{4}\right)$ for all $x \in C, y \rightarrow F(x, y)$ is convex and lower semi-continuous.

Given $r>0$, define the operator $J_{r}^{F}: H \rightarrow C$, the resolvent of $F$, by

$$
J_{r}^{F}(x)=\left\{z \in C: F(z, y)+\frac{1}{r}\langle y-z, z-x\rangle \geq 0, \forall y \in C\right\}
$$

Then,

(1) $J_{r}^{F}$ is single valued,

(2) $J_{r}^{F}$ is firmly nonexpansive, i.e, $\left\|J_{r}^{F} x-J_{r}^{F} y\right\|^{2} \leq\left\langle J_{r}^{F} x-J_{r}^{F} y, x-y\right\rangle$ for all $x, y \in H$,

(3) $\operatorname{Fix}\left(J_{r}^{F}\right)=\operatorname{EP}(F)$,

(4) $\mathrm{EP}(F)$ is closed and convex.

Let $T_{1}, T_{2}, \ldots$ be an infinite family of mappings of $C$ into itself and let $\lambda_{1}, \lambda_{2}, \ldots$ be a real numbers such that $0 \leq \lambda_{i}<1$ for every $i \in \mathbb{N}$. For any $n \in \mathbb{N}$, define a mapping $W_{n}$ of $C$ into $C$ as follows:

$$
\begin{aligned}
& U_{n, n+1}=I \\
& U_{n, n}=\lambda_{n} T_{n} U_{n, n+1}+\left(1-\lambda_{n}\right) I, \\
& U_{n, n-1}=\lambda_{n-1} T_{n-1} U_{n, n}+\left(1-\lambda_{n-1}\right) I, \\
& \vdots \\
& U_{n, k}=\lambda_{k} T_{k} U_{n, k+1}+\left(1-\lambda_{k}\right) I, \\
& U_{n, k-1}=\lambda_{k-1} T_{k-1} U_{n, k}+\left(1-\lambda_{k-1}\right) I \\
& \vdots \\
& U_{n, 2}=\lambda_{2} T_{2} U_{n, 3}+\left(1-\lambda_{2}\right) I, \\
& W_{n}=U_{n, 1}=\lambda_{1} T_{1} U_{n, 2}+\left(1-\lambda_{1}\right) I .
\end{aligned}
$$

Such a mapping $W_{n}$ is called the $W$-mapping generated by $T_{1}, T_{2}, \ldots, T_{n}$ and $\lambda_{1}, \lambda_{2}$, ..., $\lambda_{n}$.

Lemma 2.6. [29]Let $C$ be a nonempty closed convex subset of a Hilbert space $H,\left\{T_{i}\right.$ : $C \rightarrow C\}$ be an infinite family of nonexpansive mappings with $\cap_{i=1}^{\infty} \operatorname{Fix}\left(T_{i}\right) \neq \emptyset,\left\{\lambda_{i}\right\}$ be a real sequence such that $0<\lambda_{i} \leq b<1, \forall i \geq 1$. Then

(1) $W_{n}$ is nonexpansive and Fix $\left(W_{n}\right)=\cap_{i=1}^{n} \operatorname{Fix}\left(T_{i}\right)$ for each $n \geq 1$, 
(2) for each $x \in C$ and for each positive integer $j$, the limit $\lim _{n \rightarrow \infty} U_{n, j}$ exists.

(3) The mapping $W: C \rightarrow C$ defined by

$$
W x:=\lim _{n \rightarrow \infty} W_{n} x=\lim _{n \rightarrow \infty} U_{n, 1} x, \quad \forall x \in C,
$$

is a nonexpansive mapping satisfying Fix $(W)=\cap_{i=1}^{\infty}$ Fix $\left(T_{i}\right)$ and it is called the $W$ mapping generated by $T_{1}, T_{2}, \ldots$ and $\lambda_{1}, \lambda_{2}, \ldots$.

Lemma 2.7. [30]Let $C$ be a nonempty closed convex subset of a Hilbert space $H,\left\{T_{i}\right.$ : $C \rightarrow C\}$ be a countable family of nonexpansive mappings with $\cap_{i=1}^{\infty} \operatorname{Fix}\left(T_{i}\right) \neq \emptyset,\left\{\lambda_{i}\right\}$ be a real sequence such that $0<\lambda_{i} \leq b<1, \forall i \geq 1$. If $D$ is any bounded subset of $C$, then

$$
\lim _{n \rightarrow \infty} \sup _{x \in D}\left\|W x-W_{n} x\right\|=0
$$

Lemma 2.8. [31] Let $\left\{a_{n}\right\}$ be a sequence of nonnegative real numbers such that

$$
a_{n+1} \leq\left(1-b_{n}\right) a_{n}+b_{n} c_{n}, \quad n \geq 0,
$$

where $\left\{b_{n}\right\}$ and $\left\{c_{n}\right\}$ are sequences of real numbers satisfying the following conditions:

(i) $\left\{b_{n}\right\} \subset[0,1], \sum_{n=0}^{\infty} b_{n}=\infty$,

(ii) either $\limsup _{n \rightarrow \infty} c_{n} \leq 0$ or $\sum_{n=0}^{\infty}\left|b_{n} c_{n}\right|<\infty$.

Then, $\lim _{n \rightarrow \infty} a_{n}=0$.

Lemma 2.9. [32]Let $(E,\langle.,\rangle$.$) be an inner product space. Then for all x, y, z \in E$ and $\alpha, \beta, \gamma, \in[0,1]$ such that $\alpha+\beta+\gamma=1$, we have

$$
\begin{aligned}
\|\alpha x+\beta y+\gamma z\|^{2}= & \alpha\|x\|^{2}+\beta\|y\|^{2}+\gamma\|z\|^{2} \\
& -\alpha \beta\|x-\gamma\|^{2}-\alpha \gamma\|x-z\|^{2}-\beta \gamma\|y-z\|^{2} .
\end{aligned}
$$

Notation Throughout the rest of this paper the open ball of radius $r$ centered at 0 is denoted by $B_{r}$. For a subset $A$ of $H$ we denote by $\overline{c o} A$ the closed convex hull of $A$. For $\varepsilon>0$ and a mapping $T: D \rightarrow H$, we let $F_{\epsilon}(T ; D)$ be the set of $\epsilon$-approximate fixed points of $T$, i.e., $F_{\epsilon}(T ; D)=\{x \in D:\|x-T x\| \leq \epsilon\}$. Weak convergence is denoted by $\rightarrow$ and strong convergence is denoted by $\rightarrow$.

\section{Strong convergence}

Theorem 3.1. Let $C$ be a nonempty closed convex subset of a real Hilbert space $H, A: C \rightarrow$ $H$ a $\beta$-inverse strongly monotone, $B: C \rightarrow H$ a $\gamma$-inverse strongly monotone, $S$ a semigroup and $\phi=\left\{T_{t}: t \in S\right\}$ be a nonexpansive semigroup from $C$ into $C$ such that $\operatorname{Fix}(\varphi)=\cap_{t \in S} \operatorname{Fix}\left(T_{t}\right) \neq \varnothing$. Let $X$ be a left invariant subspace of $B(S)$ such that $1 \in X$, and the function $t \rightarrow\left\langle T_{t} x, y\right\rangle$ is an element of $X$ for each $x \in C$ and $y \in H,\left\{\mu_{n}\right\}$ a left regular sequence of means on $X$ such that $\lim _{n \rightarrow \infty}\left\|\mu_{n+1}-\mu_{n}\right\|=0$. Let $\mathcal{J}=\left\{F_{k}: k=1,2, \ldots, M\right\}$ be a finite family of bi-functions from $C \times C$ into $\mathbb{R}$ which satisfy $\left(A_{1}\right)-\left(A_{4}\right)$ and $\left\{T_{i}\right\}_{i=1}^{\infty}$ an infinite family of nonexpansive mappings of $C$ into $C$ such that $T_{i}(\operatorname{Fix}(\varphi) \cap \operatorname{EP}(\mathcal{J})) \subset \operatorname{Fix}(\varphi)$ for each $i \in \mathbb{N}$ and $\mathcal{F}=\cap_{i=1}^{\infty} \operatorname{Fix}\left(T_{i}\right) \cap \operatorname{Fix}(\varphi) \cap \operatorname{EP}(\mathcal{J}) \cap \operatorname{VI}(C, A) \cap \operatorname{VI}(C, B) \neq \emptyset$. Let $\left\{\alpha_{n}\right\},\left\{\beta_{n}\right\},\left\{\gamma_{n}\right\}$ and $\left\{\eta_{n}\right\}$ be a sequences in $(0,1)$. Let $\left\{\zeta_{n}\right\}$ a sequence in $(0,2 \beta),\left\{\delta_{n}\right\}$ a sequence in $(0,2 \gamma),\left\{r_{k, n}\right\}_{k=1}^{M}$ be sequences in $(0, \infty)$ and $\left\{\lambda_{n}\right\}$ a sequence of real numbers such that $0<\lambda_{n} \leq b<1$. Assume that, 
$\left(\mathrm{B}_{1}\right) \lim _{n \rightarrow \infty} \eta_{n}=\eta \in(0,1), \lim _{n \rightarrow \infty} \alpha_{n}=0$ and $\sum_{n=1}^{\infty} \alpha_{n}=\infty$,

$\left(\mathrm{B}_{2}\right) 0<\lim \inf _{n \rightarrow \infty} \beta_{n} \leq \lim \sup _{n \rightarrow \infty} \beta_{n}<1$,

$\left(\mathrm{B}_{3}\right) \alpha_{n}+\beta_{n}+\gamma_{n}=1$,

(B) $\lim _{n \rightarrow \infty}\left|\zeta_{n+1}-\zeta_{n}\right|=\lim _{n \rightarrow \infty}\left|\delta_{n+1}-\delta_{n}\right|=0$,

$\left(\mathrm{B}_{5}\right) \lim \inf _{n \rightarrow \infty} r_{k, n}>0$ and $\lim _{n \rightarrow \infty}\left(r_{k, n+1}-r_{k, n}\right)=0$ for $k \in\{1,2, \ldots, M\}$.

Let $f$ be a contraction of $C$ into itself with coefficient $\alpha \in(0,1)$ and given $x_{1} \in C$ arbitrarily. If the sequences $\left\{x_{n}\right\},\left\{y_{n}\right\}$ and $\left\{z_{n}\right\}$ are generated iteratively by $x_{1} \in C$ and

$$
\left\{\begin{array}{l}
z_{n}=J_{r_{M}, n}^{F_{M}} \ldots J_{r_{2}, n}^{F_{2}} J_{r_{1}, n}^{F_{1}} x_{n}, \\
y_{n}=\eta_{n} P_{C}\left(z_{n}-\zeta_{n} A z_{n}\right)+\left(1-\eta_{n}\right) P_{C}\left(z_{n}-\delta_{n} B z_{n}\right), \\
x_{n+1}=\alpha_{n} f\left(T_{\mu_{n}} W_{n} y_{n}\right)+\beta_{n} x_{n}+\gamma_{n} T_{\mu_{n}} W_{n} y_{n}, \quad n \geq 1,
\end{array}\right.
$$

then, the sequences $\left\{x_{n}\right\},\left\{y_{n}\right\}$ and $\left\{J_{r_{k, n}}^{F_{k}} x_{n}\right\}_{k=1}^{M}$ converge strongly to $x^{*} \in \mathcal{F}$, which is the unique solution of the system of variational inequalities:

$$
\left\{\begin{array}{l}
\left\langle f\left(x^{*}\right)-x^{*}, x^{*}-y\right\rangle \geq 0, \quad \forall y \in \mathcal{F}, \\
\left\langle B x^{*}, y-x^{*}\right\rangle \geq 0 \quad \forall y \in C \\
\left\langle A x^{*}, y-x^{*}\right\rangle \geq 0 \quad \forall y \in C .
\end{array}\right.
$$

Proof. Since $A$ is a $\beta$-inverse strongly monotone map, for any $x, y \in C$, we have

$$
\begin{aligned}
\| & \left(I-\zeta_{n} A\right) x-\left(I-\zeta_{n} A\right) y \|^{2} \\
& =\left\|(x-y)-\zeta_{n}(A x-A y)\right\|^{2} \\
& =\|\mid x-y\|^{2}-2 \zeta_{n}\langle x-y, A x-A y\rangle+\zeta_{n}^{2}\|A x-A y\|^{2} \\
& \leq\|x-y\|^{2}-2 \zeta_{n} \beta\|A x-A y\|^{2}+\zeta_{n}^{2}\|A x-A y\|^{2} \\
& =\|x-y\|^{2}+\zeta_{n}\left(\zeta_{n}-2 \beta\right)\|A x-A y\|^{2} \\
& \leq\|x-y\|^{2}
\end{aligned}
$$

It follows that

$$
\left\|\left(I-\zeta_{n} A\right) x-\left(I-\zeta_{n} A\right) y\right\| \leq\|x-y\| .
$$

Since $B$ is a $\beta$-inverse strongly monotone map, repeating the same argument as above, we can deduce that

$$
\left\|\left(I-\delta_{n} B\right) x-\left(I-\delta_{n} B\right) y\right\| \leq\|x-y\| .
$$

Let $p \in \mathcal{F}$, in the context of the variational inequality problem the characterization of projection (9) implies that $p=P_{C}\left(p-\zeta_{n} A p\right)$ and $p=P_{C}\left(p-\delta_{n} B p\right)$. Using (12) and (13), we get

$$
\begin{aligned}
\left\|y_{n}-p\right\|= & \left\|\eta_{n} P_{C}\left(z_{n}-\zeta_{n} A z_{n}\right)+\left(1-\eta_{n}\right) P_{C}\left(z_{n}-\delta_{n} B z_{n}\right)-p\right\| \\
= & \| \eta_{n}\left[P_{C}\left(z_{n}-\zeta_{n} A z_{n}\right)-P_{C}\left(p-\zeta_{n} A p\right)\right] \\
& +\left(1-\eta_{n}\right)\left[P_{C}\left(z_{n}-\delta_{n} B z_{n}\right)-P_{C}\left(p-\delta_{n} B p\right)\right] \| \\
\leq & \eta_{n}\left\|P_{C}\left(z_{n}-\zeta_{n} A z_{n}\right)-P_{C}\left(p-\zeta_{n} A p\right)\right\| \\
& +\left(1-\eta_{n}\right)\left\|P_{C}\left(z_{n}-\delta_{n} B z_{n}\right)-P_{C}\left(p-\delta_{n} B p\right)\right\| \\
\leq & \eta_{n}\left\|z_{n}-p\right\|+\left(1-\eta_{n}\right)\left\|z_{n}-p\right\|=\left\|z_{n}-p\right\| .
\end{aligned}
$$


By taking $v_{n}=P_{C}\left(z_{n}-\zeta_{n} A z_{n}\right), w_{n}=P_{C}\left(z_{n}-\delta_{n} B z_{n}\right)$ and $\mathcal{J}_{n}^{k}=J_{r_{k, n}}^{F_{k}} \cdots J_{r_{2, n}, r_{1, n}}^{F_{2}} J_{r_{1}}^{F_{1}}$ for $k \in\{1$, $2, \ldots, M\}$ and $\mathcal{J}_{n}^{0}=I$ for all $n \in \mathbb{N}$, we shall equivalently write scheme (11) as follows:

$$
\begin{aligned}
& z_{n}=\mathcal{J}_{n}^{M} x_{n} \\
& y_{n}=\eta_{n} v_{n}+\left(1-\eta_{n}\right) w_{n}, \\
& x_{n+1}=\alpha_{n} f\left(T_{\mu_{n}} W_{n} y_{n}\right)+\beta_{n} x_{n}+\gamma_{n} T_{\mu_{n}} W_{n} y_{n}, \quad n \geq 1 .
\end{aligned}
$$

We shall divide the proof into several steps.

Step 1 . The sequence $\left\{x_{n}\right\}$ is bounded.

Proof of Step 1. Let $p \in \mathcal{F}$. Since for each $k \in\{1,2, \ldots, M\}, J_{r_{k, n}}^{F_{k}}$ is nonexpansive we have

$$
\left\|\mathcal{J}_{n}^{k} x_{n}-p\right\|=\left\|\mathcal{J}_{n}^{k} x_{n}-\mathcal{J}_{n}^{k} p\right\| \leq\left\|x_{n}-p\right\|, \quad \forall k \in\{1,2, \ldots, M\} .
$$

Thus, by Lemmas 2.1, 2.5 and (14), we have

$$
\begin{aligned}
& \left\|x_{n+1}-p\right\| \\
& \leq \alpha_{n}\left\|f\left(T_{\mu_{n}} W_{n} y_{n}\right)-p\right\|+\beta_{n}\left\|x_{n}-p\right\|+\gamma_{n}\left\|T_{\mu_{n}} W_{n} \mathcal{J}_{n}^{\mathrm{M}} y_{n}-p\right\| \\
& \leq \alpha_{n}\left[\left\|f\left(T_{\mu_{n}} W_{n} y_{n}\right)-f(p)\right\|+\|f(p)-p\|\right]+\beta_{n}\left\|x_{n}-p\right\|+\gamma_{n}\left\|y_{n}-p\right\| \\
& \leq \alpha_{n} \alpha\left\|x_{n}-p\right\|+\alpha_{n}\|f(p)-p\|+\left(\beta_{n}+\gamma_{n}\right)\left\|x_{n}-p\right\| \\
& =\left[1-\alpha_{n}(1-\alpha)\right]\left\|x_{n}-p\right\|+\alpha_{n}\|f(p)-p\| \\
& \leq \max \left\{\left\|x_{n}-p\right\|, \frac{1}{1-\alpha}\|f(p)-p\|\right\} .
\end{aligned}
$$

By induction,

$$
\left\|x_{n}-p\right\| \leq \max \left\{\left\|x_{1}-p\right\|, \frac{1}{1-\alpha}\|f(p)-p\|\right\}, \quad n \geq 1 .
$$

Step 2. Let $\left\{u_{n}\right\}$ be a bounded sequence in $H$. Then

$$
\lim _{n \rightarrow \infty}\left\|\mathcal{J}_{n+1}^{k} u_{n}-\mathcal{J}_{n}^{k} u_{n}\right\|=0,
$$

for every $k \in\{1,2, \ldots, M\}$.

Proof of Step 2. This assertion is proved in [27, Step 2].

Step 3. Let $\left\{u_{n}\right\}$ be a bounded sequence in $H$. Then

$$
\lim _{n \rightarrow \infty}\left\|W_{n+1} u_{n}-W_{n} u_{n}\right\|=0 \text { and } \lim _{n \rightarrow \infty}\left\|T_{\mu_{n+1}} u_{n}-T_{\mu_{n}} u_{n}\right\|=0 .
$$

This assertion is proved in [21, Step 3].

Step 4. $\lim _{n \rightarrow \infty}\left\|x_{n+1}-x_{n}\right\|=0$.

Proof of Step 4. Setting $x_{n+1}=\beta_{n} x_{n}+\left(1-\beta_{n}\right) t_{n}$ for all $n \geq 1$, we have

$$
\begin{aligned}
t_{n+1} & -t_{n} \\
= & \frac{1}{1-\beta_{n+1}}\left[x_{n+2}-\beta_{n+1} x_{n+1}\right]-\frac{1}{1-\beta_{n}}\left[x_{n+1}-\beta_{n} x_{n}\right] \\
= & \frac{\alpha_{n+1}}{1-\beta_{n+1}}\left[f\left(T_{\mu_{n+1}} W_{n+1} y_{n+1}\right)-f\left(T_{\mu_{n}} W_{n} y_{n}\right)\right]+\left(\frac{\alpha_{n+1}}{1-\beta_{n+1}}-\frac{\alpha_{n}}{1-\beta_{n}}\right) f\left(T_{\mu_{n}} W_{n} y_{n}\right) \\
& +\frac{\gamma_{n+1}}{1-\beta_{n+1}}\left[T_{\mu_{n+1}} W_{n+1} y_{n+1}-T_{\mu_{n}} W_{n} y_{n}\right] \\
& +\left[\frac{\gamma_{n+1}}{1-\beta_{n+1}}-\frac{\gamma_{n}}{1-\beta_{n}}\right] T_{\mu_{n}} W_{n} y_{n} .
\end{aligned}
$$


Therefore, we have

$$
\begin{aligned}
& \left\|t_{n+1}-t_{n}\right\| \\
& \quad \leq \frac{\alpha_{n+1}}{1-\beta_{n+1}}\left[\alpha\left\|T_{\mu_{n+1}} W_{n+1} y_{n+1}-T_{\mu_{n}} W_{n} y_{n}\right\|+\left\|T_{\mu_{n+1}} W_{n+1} y_{n+1}-T_{\mu_{n}} W_{n} y_{n}\right\|\right] \\
& \quad+\left|\frac{\alpha_{n+1}}{1-\beta_{n+1}}-\frac{\alpha_{n}}{1-\beta_{n}}\right|\left[\left\|f\left(T_{\mu_{n}} W_{n} y_{n}\right)\right\|+\left\|T_{\mu_{n}} W_{n} y_{n}\right\|\right] \\
& \quad+\left\|T_{\mu_{n+1}} W_{n+1} y_{n+1}-T_{\mu_{n}} W_{n} y_{n}\right\| .
\end{aligned}
$$

On the other hand

$$
\begin{aligned}
& \left\|T_{\mu_{n+1}} W_{n+1} y_{n+1}-T_{\mu_{n}} W_{n} y_{n}\right\| \\
& \leq \quad\left\|T_{\mu_{n+1}} W_{n+1} y_{n+1}-T_{\mu_{n+1}} W_{n+1} y_{n}\right\| \\
& \quad+\left\|T_{\mu_{n+1}} W_{n+1} y_{n}-T_{\mu_{n+1}} W_{n} y_{n}\right\| \\
& \quad+\left\|T_{\mu_{n+1}} W_{n} y_{n}-T_{\mu_{n}} W_{n} y_{n}\right\| \\
& \leq\left\|y_{n+1}-y_{n}\right\|+\left\|W_{n+1} y_{n}-W_{n} y_{n}\right\|+\left\|T_{\mu_{n+1}} W_{n} y_{n}-T_{\mu_{n}} W_{n} y_{n}\right\|
\end{aligned}
$$

Observing that $z_{n}=\mathcal{J}_{n}^{M} x_{n}, z_{n+1}=\mathcal{J}_{n+1}^{M} x_{n+1}$ and $\mathcal{J}_{n}^{M} x_{n}=J_{r_{M}, n}^{F_{M}} \mathcal{J}_{n}^{M-1} x_{n}$ we get

$$
\frac{1}{r_{M, n}}\left\langle y-z_{n}, z_{n}-\mathcal{J}_{n}^{M-1} x_{n}\right\rangle+F_{M}\left(z_{n}, y\right) \geq 0, \quad \forall y \in C,
$$

and

$$
\frac{1}{r_{M, n+1}}\left\langle y-z_{n+1}, z_{n+1}-\mathcal{J}_{n+1}^{M-1} x_{n+1}\right\rangle+F_{M}\left(z_{n+1}, y\right) \geq 0, \quad \forall y \in C,
$$

Take $y=z_{n+1}$ in (17) and $y=z_{n}$ in (18), by using $\left(A_{2}\right)$, it follows that

$$
\left\langle z_{n+1}-z_{n}, z_{n}-\mathcal{J}_{n}^{M-1} x_{n}-\frac{r_{M, n}}{r_{M, n+1}}\left(z_{n+1}-\mathcal{J}_{n+1}^{M-1} x_{n+1}\right)\right\rangle \geq 0,
$$

and hence

$$
\begin{aligned}
\left\langle z_{n+1}-z_{n},\right. & z_{n}-\mathcal{J}_{n}^{M-1} x_{n}-z_{n+1}+\mathcal{J}_{n+1}^{M-1} x_{n+1} \\
& \left.+\left(1-\frac{r_{M, n}}{r_{M, n+1}}\right)\left(z_{n+1}-\mathcal{J}_{n+1}^{M-1} x_{n+1}\right)\right\rangle \geq 0
\end{aligned}
$$

Thus, we have

$$
\begin{aligned}
& \left\|z_{n+1}-z_{n}\right\| \\
& \leq\left\|\mathcal{J}_{n+1}^{M-1} x_{n+1}-\mathcal{J}_{n}^{M-1} x_{n}\right\|+\left|1-\frac{r_{M, n}}{r_{M, n+1}}\right|\left\|z_{n+1}-\mathcal{J}_{n+1}^{M-1} x_{n+1}\right\| \\
& \leq\left\|\mathcal{J}_{n+1}^{M-1} x_{n+1}-\mathcal{J}_{n+1}^{M-1} x_{n}\right\|+\left\|\mathcal{J}_{n+1}^{M-1} x_{n}-\mathcal{J}_{n}^{M-1} x_{n}\right\| \\
& \quad+\left|1-\frac{r_{M, n}}{r_{M, n+1}}\right|\left\|z_{n+1}-\mathcal{J}_{n+1}^{M-1} x_{n+1}\right\| \\
& \leq \mid\left\|x_{n+1}-x_{n}\right\|+\left\|\mathcal{J}_{n+1}^{M-1} x_{n}-\mathcal{J}_{n}^{M-1} x_{n}\right\| \\
& \quad+\left|1-\frac{r_{M, n}}{r_{M, n+1}}\right|\left\|z_{n+1}-\mathcal{J}_{n+1}^{M-1} x_{n+1}\right\| .
\end{aligned}
$$

Since $v_{n}=P_{C}\left(z_{n}-\zeta_{n} A z_{n}\right)$ and $w_{n}=P_{C}\left(z_{n}-\delta_{n} B z_{n}\right)$, it follows from the definition of $\left\{y_{n}\right\}$ that 
Peri Fixed Point Theory and Applications 2012, 2012:99

Page 11 of 22

http://www.fixedpointtheoryandapplications.com/content/2012/1/99

$$
\begin{aligned}
&\left\|y_{n+1}-y_{n}\right\| \\
&=\left\|\eta_{n+1} v_{n+1}+\left(1-\eta_{n+1}\right) w_{n+1}-\eta_{n} v_{n}-\left(1-\eta_{n}\right) w_{n}\right\| \\
&= \| \eta_{n+1}\left(v_{n+1}-v_{n}\right)+\left(\eta_{n+1}-\eta_{n}\right) v_{n}+\left(1-\eta_{n+1}\right) w_{n+1} \\
&-\left(1-\eta_{n+1}\right) w_{n}+\left(\eta_{n}-\eta_{n+1}\right) w_{n} \| \\
& \leq \eta_{n+1}\left\|v_{n+1}-v_{n}\right\|+\left|\eta_{n+1}-\eta_{n}\right|\left(\left\|v_{n}\right\|+\left\|w_{n}\right\|\right) \\
&+\left(1-\eta_{n+1}\right)\left\|w_{n+1}-w_{n}\right\| \\
&= \eta_{n+1}\left\|P_{C}\left(z_{n+1}-\zeta_{n+1} A z_{n+1}\right)-P_{C}\left(z_{n}-\zeta_{n} A z_{n}\right)\right\| \\
&+\left|\eta_{n+1}-\eta_{n}\right|\left(\left\|v_{n}\right\|+\left\|w_{n}\right\|\right) \\
&+\left(1-\eta_{n+1}\right)\left\|P_{C}\left(z_{n+1}-\delta_{n+1} B z_{n+1}\right)-P_{C}\left(z_{n}-\delta_{n} B z_{n}\right)\right\| \\
&= \eta_{n+1} \| P_{C}\left(z_{n+1}-\zeta_{n+1} A z_{n+1}\right)-P_{C}\left(z_{n}-\zeta_{n+1} A z_{n}\right) \\
&+P_{C}\left(z_{n}-\zeta_{n+1} A z_{n}\right)-P_{C}\left(z_{n}-\zeta_{n} A z_{n}\right) \| \\
&+\left|\eta_{n+1}-\eta_{n}\right|\left(\left\|v_{n}\right\|+\left\|w_{n}\right\|\right) \\
&+\left(1-\eta_{n+1}\right) \| P_{C}\left(z_{n+1}-\delta_{n+1} B z_{n+1}\right)-P_{C}\left(z_{n}-\delta_{n+1} B z_{n}\right) \\
&+P_{C}\left(z_{n}-\delta_{n+1} B z_{n}\right)-P_{C}\left(z_{n}-\delta_{n} B z_{n}\right) \| \\
& \leq \eta_{n+1}\left\|z_{n+1}-z_{n}\right\|+\eta_{n+1}\left|\zeta_{n+1}-\zeta_{n}\right|\left\|A z_{n}\right\| \\
&+\left|\eta_{n+1}-\eta_{n}\right|\left(\left\|v_{n}\right\|+\left\|w_{n}\right\|\right)+\left(1-\eta_{n+1}\right)\left\|z_{n+1}-z_{n}\right\| \\
&+\left(1-\eta_{n+1}\right)\left|\delta_{n+1}-\delta_{n}\right|\left\|B z_{n}\right\| \\
& \leq\left\|z_{n+1}-z_{n}\right\|+\eta_{n+1}\left|\zeta_{n+1}-\zeta_{n}\right|\left\|A z_{n}\right\| \\
&+\left|\eta_{n+1}-\eta_{n}\right|\left(\left\|v_{n}\right\|+\left\|w_{n}\right\|\right)+\left|\delta_{n+1}-\delta_{n}\right|\left\|B z_{n}\right\| .
\end{aligned}
$$

Therefore,

$$
\begin{aligned}
& \left\|t_{n+1}-t_{n}\right\|-\left\|x_{n+1}-x_{n}\right\| \\
& \leq \frac{\alpha_{n+1}}{1-\beta_{n+1}}\left[\left\|y_{n+1}-y_{n}\right\|+\left\|T_{\mu_{n+1}} W_{n+1} y_{n+1}-T_{\mu_{n}} W_{n} y_{n}\right\|\right] \\
& \quad+\left|\frac{\alpha_{n+1}}{1-\beta_{n+1}}-\frac{\alpha_{n}}{1-\beta_{n}}\right|\left[\left\|f\left(T_{\mu_{n}} W_{n} y_{n}\right)\right\|+\left\|T_{\mu_{n}} W_{n} y_{n}\right\|\right] \\
& \quad+\left\|\mathcal{J}_{n+1}^{M-1} x_{n}-\mathcal{J}_{n}^{M-1} x_{n}\right\|+\left|1-\frac{r_{M, n}}{r_{M, n+1}}\right|\left\|z_{n+1}-\mathcal{J}_{n+1}^{M-1} x_{n+1}\right\| \\
& \quad+\eta_{n+1}\left|\zeta_{n+1}-\zeta_{n}\right|\left\|A z_{n}\right\|+\left|\eta_{n+1}-\eta_{n}\right|\left(\left\|v_{n}\right\|+\left\|w_{n}\right\|\right) \\
& \quad+\left|\delta_{n+1}-\delta_{n}\right|\left\|B z_{n}\right\|+\left\|W_{n+1} y_{n}-W_{n} y_{n}\right\|+\left\|T_{\mu_{n+1}} W_{n} y_{n}-T_{\mu_{n}} W_{n} y_{n}\right\| .
\end{aligned}
$$

This together with conditions $\left(B_{1}\right),\left(B_{4}\right)$, Steps 2 and 3 imply that

$$
\limsup _{n \rightarrow \infty}\left(\left\|t_{n+1}-t_{n}\right\|-\left\|x_{n+1}-x_{n}\right\|\right) \leq 0 .
$$

Hence by Lemma 2.4, we obtain $\lim _{n \rightarrow \infty}\left\|t_{n}-x_{n}\right\|=0$. Consequently,

$$
\lim _{n \rightarrow \infty}\left\|x_{n+1}-x_{n}\right\|=\left(1-\beta_{n}\right)\left\|t_{n}-x_{n}\right\|=0 .
$$

Step 5. $\lim _{n \rightarrow \infty}\left\|\mathcal{J}_{n}^{k+1} x_{n}-\mathcal{J}_{n}^{k} x_{n}\right\|=0, \forall k \in\{0,1,2, \ldots, M-1\}$.

Proof of Step 5. Let $p \in \mathcal{F}$ and $k \in\{1,2, \ldots, M-1\}$. Since $J_{r_{k+1, n}}^{F_{k+1}}$ is firmly nonexpansine, we obtain

$$
\begin{aligned}
& \left\|\mathcal{J}_{n}^{k+1} x_{n}-p\right\|^{2} \\
& \quad=\left\|J_{r_{k+1, n}}^{F_{k+1}} \mathcal{J}_{n}^{k} x_{n}-J_{r_{k+1, n}}^{F_{k+1}} p\right\|^{2}=\left\langle J_{r_{k+1, n}}^{F_{k+1}} \mathcal{J}_{n}^{k} x_{n}-p, \mathcal{J}_{n}^{k} x_{n}-p\right\rangle \\
& \quad=\frac{1}{2}\left[\left\|J_{r_{k+1, n}}^{F_{k+1}} \mathcal{J}_{n}^{k} x_{n}-p\right\|^{2}+\left\|\mathcal{J}_{n}^{k} x_{n}-p\right\|^{2}-\left\|J_{r_{k+1, n}}^{F_{k+1}} \mathcal{J}_{n}^{k} x_{n}-\mathcal{J}_{n}^{k} x_{n}\right\|^{2}\right] .
\end{aligned}
$$


It follows that

$$
\left\|\mathcal{J}_{n}^{k+1} x_{n}-p\right\|^{2} \leq\left\|x_{n}-p\right\|^{2}-\left\|\mathcal{J}_{n}^{k+1} x_{n}-\mathcal{J}_{n}^{k} x_{n}\right\|^{2} .
$$

Using Lemma 2.9, (14) and (19), we obtain

$$
\begin{aligned}
\| & x_{n+1}-p \|^{2} \\
\leq & \alpha_{n}\left\|f\left(T_{\mu_{n}} W_{n} y_{n}\right)-p\right\|^{2}+\beta_{n}\left\|x_{n}-p\right\|^{2}+\gamma_{n}\left\|T_{\mu_{n}} W_{n} y_{n}-p\right\|^{2} \\
\leq & \alpha_{n}\left\|f\left(T_{\mu_{n}} W_{n} y_{n}\right)-p\right\|^{2}+\beta_{n}\left\|x_{n}-p\right\|^{2}+\gamma_{n}\left\|y_{n}-p\right\|^{2} \\
\leq & \alpha_{n}\left\|f\left(T_{\mu_{n}} W_{n} y_{n}\right)-p\right\|^{2}+\beta_{n}\left\|x_{n}-p\right\|^{2}+\gamma_{n}\left\|z_{n}-p\right\|^{2} \\
= & \alpha_{n}\left\|f\left(T_{\mu_{n}} W_{n} y_{n}\right)-p\right\|^{2}+\beta_{n}\left\|x_{n}-p\right\|^{2}+\gamma_{n}\left\|\mathcal{J}_{n}^{M} x_{n}-p\right\|^{2} \\
= & \alpha_{n}\left\|f\left(T_{\mu_{n}} W_{n} y_{n}\right)-p\right\|^{2}+\beta_{n}\left\|x_{n}-p\right\|^{2} \\
& +\gamma_{n}\left\|J_{r_{M}, n}^{F_{M}} \ldots J_{r_{k+2}, n}^{F_{k+2}} \mathcal{J}_{n}^{k+1} y_{n}-J_{r_{M}, n}^{F_{M}} \cdots J_{r_{k+2}, n}^{F_{k+2}} p\right\|^{2} \\
\leq & \alpha_{n}\left\|f\left(T_{\mu_{n}} W_{n} y_{n}\right)-p\right\|^{2}+\beta_{n}\left\|x_{n}-p\right\|^{2}+\gamma_{n}\left\|\mathcal{J}_{n}^{k+1} y_{n}-p\right\|^{2} \\
\leq & \alpha_{n}\left\|f\left(T_{\mu_{n}} W_{n} y_{n}\right)-p\right\|^{2}+\beta_{n}\left\|x_{n}-p\right\|^{2}+\gamma_{n}\left\|\mathcal{J}_{n}^{k+1} x_{n}-p\right\|^{2} \\
\leq & \alpha_{n}\left\|f\left(T_{\mu_{n}} W_{n} y_{n}\right)-p\right\|^{2}+\beta_{n}\left\|x_{n}-p\right\|^{2} \\
& +\gamma_{n}\left[\left\|x_{n}-p\right\|^{2}-\left\|\mathcal{J}_{n}^{k+1} x_{n}-\mathcal{J}_{n}^{k} x_{n}\right\|\right] .
\end{aligned}
$$

Then, we have

$$
\begin{aligned}
\gamma_{n} & \left\|\mathcal{J}_{n}^{k+1} x_{n}-\mathcal{J}_{n}^{k} x_{n}\right\|^{2} \\
\leq & \alpha_{n}\left\|f\left(T_{\mu_{n}} W_{n} y_{n}\right)-p\right\|^{2}+\beta_{n}\left\|x_{n}-p\right\|^{2} \\
& +\left(1-\alpha_{n}-\beta_{n}\right)\left\|x_{n}-p\right\|^{2}-\left\|x_{n+1}-p\right\|^{2} \\
= & \alpha_{n}\left[\left\|f\left(T_{\mu_{n}} W_{n} y_{n}\right)-p\right\|^{2}-\left\|x_{n}-p\right\|^{2}\right]+\left\|x_{n}-p\right\|^{2}-\left\|x_{n+1}-p\right\|^{2} \\
\leq & \alpha_{n}\left[\left\|f\left(T_{\mu_{n}} W_{n} y_{n}\right)-p\right\|^{2}-\left\|x_{n}-p\right\|^{2}\right] \\
& +\left\|x_{n}-x_{n+1}\right\|\left[\left\|x_{n}-p\right\|+\left\|x_{n+1}-p\right\|\right] .
\end{aligned}
$$

It is easily seen that $\lim \inf _{n \rightarrow \infty} \gamma_{n}>0$. So we have

$$
\lim _{n \rightarrow \infty}\left\|\mathcal{J}_{n}^{k+1} x_{n}-\mathcal{J}_{n}^{k} x_{n}\right\|=0 .
$$

Step 6. $\lim _{n \rightarrow \infty}\left\|x_{n}-T_{\mu_{n}} W_{n} \mathcal{J}_{n}^{M} y_{n}\right\|=0$.

Proof of Step 6. Observe that

$$
\begin{aligned}
&\left\|x_{n}-T_{\mu_{n}} W_{n} \mathcal{J}_{n}^{M} y_{n}\right\| \\
& \leq \quad\left\|x_{n}-x_{n+1}\right\|+\left\|x_{n+1}-T_{\mu_{n}} W_{n} \mathcal{J}_{n}^{M} y_{n}\right\| \\
&=\left\|x_{n}-x_{n+1}\right\|+\| \alpha_{n}\left[f\left(T_{\mu_{n}} W_{n} y_{n}\right)-T_{\mu_{n}} W_{n} \mathcal{J}_{n}^{M} y_{n}\right] \\
&+\beta_{n}\left[x_{n}-T_{\mu_{n}} W_{n} \mathcal{J}_{n}^{M} y_{n}\right] \| \\
& \leq\left\|x_{n}-x_{n+1}\right\|+\alpha_{n}\left[\left\|f\left(T_{\mu_{n}} W_{n} y_{n}\right)\right\|+\left\|T_{\mu_{n}} W_{n} \mathcal{J}_{n}^{M} y_{n}\right\|\right] \\
&+\beta_{n}\left\|x_{n}-T_{\mu_{n}} W_{n} \mathcal{J}_{n}^{M} y_{n}\right\|,
\end{aligned}
$$

hence

$$
\begin{aligned}
\| x_{n} & -T_{\mu_{n}} W_{n} \mathcal{J}_{n}^{M} y_{n} \| \\
& \leq \frac{1}{1-\beta_{n}}\left\|x_{n+1}-x_{n}\right\|+\frac{\alpha_{n}}{1-\beta_{n}}\left[\left\|f\left(T_{\mu_{n}} W_{n} y_{n}\right)\right\|+\left\|T_{\mu_{n}} W_{n} \mathcal{J}_{n}^{M} y_{n}\right\|\right] .
\end{aligned}
$$


It follows from conditions $\left(B_{1}\right),\left(B_{2}\right)$ and Step 4, that

$$
\lim _{n \rightarrow \infty}\left\|x_{n}-T_{\mu_{n}} W_{n} \mathcal{J}_{n}^{M} y_{n}\right\|=0 .
$$

Step 7. $\lim _{n \rightarrow \infty}\left\|x_{n}-T_{t} x_{n}\right\|=0$, for all $t \in S$.

Proof of Step 7. Let $p \in \mathcal{F}$ and set $M_{0}=\max \left\{\left\|x_{1}-p\right\|, \frac{1}{1-\alpha}\|f(p)-p\|\right\}$ and $D=\{y$ $\left.\in H:\|y-p\| \leq M_{0}\right\}$, we remark that $D$ is bounded closed convex set, $\left\{y_{n}\right\} \subset D$ and it is invariant under $\left\{J_{r_{k, n}}^{F_{k}}: k=1,2, \ldots, M, \forall n \in \mathbb{N}\right\}, \phi$ and $W_{n}$ for all $n \in \mathbb{N}$. We will show that

$$
\limsup _{n \rightarrow \infty} \sup _{\gamma \in D}\left\|T_{\mu_{n}} y-T_{t} T_{\mu_{n}} y\right\|=0, \quad \forall t \in S
$$

Let $\epsilon>0$. By [33, Theorem 1.2], there exists $\delta>0$ such that

$$
\overline{c o} F_{\delta}\left(T_{t} ; D\right)+B_{\delta} \subset F_{\varepsilon}\left(T_{t} ; D\right), \quad \forall t \in S .
$$

Also by [33, Corollary 1.1], there exists a natural number $N$ such that

$$
\left\|\frac{1}{N+1} \sum_{i=0}^{N} T_{t^{i} s} y-T_{t}\left(\frac{1}{N+1} \sum_{i=0}^{N} T_{t^{i} s} y\right)\right\| \leq \delta,
$$

for all $t, s \in S$ and $y \in D$. Let $t \in S$. Since $\left\{\mu_{n}\right\}$ is strongly left regular, there exists $N_{0}$ $\in \mathbb{N}$ such that $\left\|\mu_{n}-l_{t^{i}}^{*} \mu_{n}\right\| \leq \frac{\delta}{\left(M_{0}+\|p\|\right)}$ for $n \geq N_{0}$ and $i=1,2, \ldots, N$. Then, we have

$$
\begin{aligned}
\sup _{\gamma \in D} & \| T_{\mu_{n}} \gamma-\int \frac{1}{N+1} \sum_{i=0}^{N} T_{t^{i} s} \gamma d \mu_{n}(s)|| \\
= & \sup _{\gamma \in D} \sup _{\|z\|=1}\left|\left\langle T_{\mu_{n}} y, z\right\rangle-\left\langle\int \frac{1}{N+1} \sum_{i=0}^{N} T_{t^{i} s} y d \mu_{n}(s), z\right\rangle\right| \\
= & \sup _{\gamma \in D} \sup _{\|z\|=1}\left|\frac{1}{N+1} \sum_{i=0}^{N}\left(\mu_{n}\right)_{s}\left\langle T_{s} y, z\right\rangle-\frac{1}{N+1} \sum_{i=0}^{N}\left(\mu_{n}\right)_{s}\left\langle T_{t^{i}} y, z\right\rangle\right| \\
\leq & \frac{1}{N+1} \sum_{i=0}^{N} \sup _{\gamma \in D} \sup _{\|z\|=1}\left|\left(\mu_{n}\right)_{s}\left\langle T_{s} y, z\right\rangle-\left(l_{t^{i}}^{*} \mu_{n}\right)_{s}\left\langle T_{s} y, z\right\rangle\right| \\
\leq & \max _{i=1,2, \ldots, N}\left\|\mu_{n}-l_{t^{\prime}}^{*} \mu_{n}\right\|\left(M_{0}+\|p\|\right) \leq \delta, \quad \forall n \geq N_{0} .
\end{aligned}
$$

By Lemma 2.1 we have

$$
\int \frac{1}{N+1} \sum_{i=0}^{N} T_{t^{i} s} \gamma d \mu_{n}(s) \in \overline{c o}\left\{\frac{1}{N+1} \sum_{i=0}^{N} T_{t^{i}}\left(T_{s} \gamma\right): s \in S\right\} .
$$

It follows from (21), (22), (23) and (24) that

$$
\begin{gathered}
T_{\mu_{n}} \gamma \in \overline{c o}\left\{\frac{1}{N+1} \sum_{i=0}^{N} T_{t^{i} s} y: s \in S\right\}+B_{\delta} \\
\subset \overline{c o} F_{\delta}\left(T_{t} ; D\right)+B_{\delta} \subset F_{\varepsilon}\left(T_{t} ; D\right),
\end{gathered}
$$


for all $y \in D$ and $n \geq N_{0}$. Therefore,

$$
\limsup _{n \rightarrow \infty} \sup _{\gamma \in D}\left\|T_{t}\left(T_{\mu_{n}} y\right)-T_{\mu_{n}} y\right\| \leq \varepsilon
$$

Since $\epsilon>0$ is arbitrary, we get (20).

Let $t \in S$ and $\epsilon>0$. Then, there exists $\delta>0$, which satisfies (21). From condition $\left(B_{1}\right)$, (20) and Step 6, there exists $N_{1} \in \mathbb{N}$ such that $\alpha_{n}<\frac{\delta}{4 M_{0}}, T_{\mu_{n}} y \in F_{\delta}\left(T_{t}, D\right)$ for all $y \in$ $D$ and $\left\|x_{n}-T_{\mu_{n}} W_{n} y_{n}\right\|<\frac{\delta}{2}$ for all $n \geq N_{1}$. We note that

$$
\begin{aligned}
\alpha_{n} & \left\|f\left(T_{\mu_{n}} W_{n} y_{n}\right)-T_{\mu_{n}} W_{n} y_{n}\right\| \\
& \leq \alpha_{n}\left[\left\|f\left(T_{\mu_{n}} W_{n} y_{n}\right)-f(p)\right\|+\|f(p)-p\|+\left\|p-T_{\mu_{n}} W_{n} y_{n}\right\|\right] \\
& \leq \alpha_{n}\left[\alpha\left\|y_{n}-p\right\|+\|f(p)-p\|+\left\|p-y_{n}\right\|\right] \leq 2 M_{0} \alpha_{n} \leq \frac{\delta}{2}
\end{aligned}
$$

for all $n \geq N_{1}$. Therefore, we have

$$
\begin{aligned}
x_{n+1}= & T_{\mu_{n}} W_{n} y_{n}+\alpha_{n}\left(f\left(T_{\mu_{n}} W_{n} y_{n}\right)-T_{\mu_{n}} W_{n} y_{n}\right) \\
& +\beta_{n}\left(x_{n}-T_{\mu_{n}} W_{n} y_{n}\right) \\
\in & F_{\delta}\left(T_{t} ; D\right)+B_{\frac{\delta}{2}}+B_{\frac{\delta}{2}} \subset F_{\delta}\left(T_{t} ; D\right)+B_{\delta} \subset F_{\varepsilon}\left(T_{t} ; D\right),
\end{aligned}
$$

for all $n \geq N_{1}$. This shows that

$$
\left\|x_{n}-T_{t} x_{n}\right\| \leq \varepsilon, \quad \forall n \geq N_{1} .
$$

Since $\epsilon>0$ is arbitrary, we get $\lim _{n \rightarrow \infty}\left\|x_{n}-T_{t}\left(x_{n}\right)\right\|=0$.

Step 8. The weak $\omega$-limit set of $\left\{x_{n}\right\}, \omega_{\omega}\left\{x_{n}\right\}$, is a subset of $\mathcal{F}$.

Proof of Step 8. Let $z \in \omega_{\omega}\left\{x_{n}\right\}$ and let $\left\{x_{n_{m}}\right\}$ be a subsequence of $\left\{x_{n}\right\}$ weakly converging to $z$, we need to show that $z \in \mathcal{F}$. Noting Step 5 , with no loss of generality, we may assume that $\mathcal{J}_{n_{m}}^{k} x_{n_{m}} \rightarrow z, \forall k \in\{1,2, \ldots, M\}$. At first, note that by $\left(A_{2}\right)$ and given $y$ $\in C$ and $k \in\{1,2, \ldots, M\}$, we have

$$
\left\langle y-\mathcal{J}_{n_{m}}^{k+1} x_{n_{m}} \frac{1}{r_{k+1, n_{m}}}\left(\mathcal{J}_{n_{m}}^{k+1} x_{n_{m}}-\mathcal{J}_{n_{m}}^{k} x_{n_{m}}\right)\right\rangle \geq F_{k+1}\left(y, \mathcal{J}_{n_{m}}^{k+1} x_{n_{m}}\right)
$$

Step 5 and condition $\left(B_{5}\right)$ imply that

$$
\frac{\mathcal{J}_{n_{m}}^{k+1} x_{n_{m}}-\mathcal{J}_{n_{m}}^{k} x_{n_{m}}}{r_{k+1, n_{m}}} \rightarrow 0
$$

Since $\mathcal{J}_{n_{m}}^{k} x_{n_{m}} \rightarrow z$, from the lower semi-continuity of $F_{k+1}$ on the second variable, we have $F_{k+1}(y, z) \leq 0$ for all $y \in C$ and for all $k \in\{0,1,2, \ldots, M-1\}$. For $t$ with $0<t \leq 1$ and $y \in C$, let $y_{t}=t y+(1-t) z$. Since $y \in C$ and $z \in C$, we have $y_{t} \in C$ and hence $F_{k}$ ${ }_{+1}\left(y_{t}, z\right) \leq 0$. So from the convexity of $F_{k+1}$ on second variable, we have

$$
0=F_{k+1}\left(y_{t}, y_{t}\right) \leq t F_{k+1}\left(y_{t}, \gamma\right)+(1-t) F_{k+1}\left(y_{t}, z\right) \leq t F_{k+1}\left(y_{t}, y\right) \leq F_{k+1}\left(y_{t}, y\right) .
$$

hence $F_{k+1}\left(y_{t}, y\right) \geq 0$. therefore, we have $F_{k+1}(z, y) \geq 0$ for all $y \in C$ and $k \in\{0,1,2$, ..., $M-1\}$. Therefore $z \in \cap_{k=1}^{M} \operatorname{EP}\left(F_{k}\right)=\operatorname{EP}(\mathcal{J})$.

Since $x_{n_{m}} \rightarrow z$, it follows by Step 7 and Lemma 2.2 that $z \in \operatorname{Fix}\left(T_{t}\right)$ for all $t \in S$. Therefore, $z \in \operatorname{Fix}(\phi)$. We will show $z \in \operatorname{Fix}(W)$. Assume $z \notin \operatorname{Fix}(W)$ Since 
$z \in \operatorname{Fix}(\varphi) \cap \operatorname{EP}(\mathcal{J})$, by our assumption, we have $T_{i} z \in \operatorname{Fix}(\phi), \forall i \in \mathbb{N}$ and then $W_{n} z \in$ $\operatorname{Fix}(\phi)$. Hence by Lemma 2.1, $T_{\mu_{n}} W_{n} z=W_{n} z$, therefore by Lemma 2.5 , we get

$$
T_{\mu_{n}} W_{n} \mathcal{J}_{n}^{M} z=W_{n} z, \quad \forall n \in \mathbb{N} .
$$

Now, by (25), Step 6, Lemma 2.6 and Opial's condition, we have

$$
\begin{aligned}
& \liminf _{n \rightarrow \infty}\left\|x_{n_{m}}-z\right\| \\
& \quad<\liminf _{n \rightarrow \infty}\left\|x_{n_{m}}-W z\right\| \\
& \leq \liminf _{n \rightarrow \infty}\left(\left\|x_{n_{m}}-T_{\mu_{n_{m}}} W_{n_{m}} \mathcal{J}_{n_{m}}^{M} x_{n_{m}}\right\|\right. \\
& \left.\quad+\left\|T_{\mu_{n_{m}}} W_{n_{m}} \mathcal{J}_{n_{m}}^{M} x_{n_{m}}-T_{\mu_{n_{m}}} W_{n_{m}} \mathcal{J}_{n_{m}}^{M} z\right\|+\left\|T_{\mu_{n_{m}}} W_{n_{m}} \mathcal{J}_{n_{m}}^{M} z-W z\right\|\right) \\
& \leq \liminf _{n \rightarrow \infty}\left(\left\|x_{n_{m}}-T_{\mu_{n_{m}}} W_{n_{m}} \mathcal{J}_{n_{m}}^{M} x_{n_{m}}\right\|+\left\|x_{n_{m}}-z\right\|+\left\|W_{n_{m}} z-W z\right\|\right) \\
& \quad \leq \liminf _{n \rightarrow \infty}\left\|x_{n_{m}}-z\right\| .
\end{aligned}
$$

This is a contradiction. So we get $z \in \operatorname{Fix}(W)=\bigcap_{i=1}^{\infty} \operatorname{Fix}\left(T_{i}\right)$.

Now, let us show that $z \in \operatorname{VI}(C, A) \cap \operatorname{VI}(C, B)$. Observe that,

$$
\begin{aligned}
\| x_{n+1}- & p \|^{2} \\
\leq & \alpha_{n}\left\|f\left(T_{\mu_{n}} W_{n} y_{n}\right)-p\right\|^{2}+\beta_{n}\left\|x_{n}-p\right\|^{2}+\gamma_{n}\left\|T_{\mu_{n}} W_{n} \mathcal{J}_{n}^{M} y_{n}-p\right\|^{2} \\
\leq & \alpha_{n}\left\|f\left(T_{\mu_{n}} W_{n} y_{n}\right)-p\right\|^{2}+\beta_{n}\left\|x_{n}-p\right\|^{2}+\gamma_{n}\left\|y_{n}-p\right\|^{2} \\
= & \alpha_{n}\left\|f\left(T_{\mu_{n}} W_{n} y_{n}\right)-p\right\|^{2}+\beta_{n}\left\|x_{n}-p\right\|^{2}+\gamma_{n} \| \eta_{n} P_{C}\left(z_{n}-\zeta_{n} A z_{n}\right) \\
& +\left(1-\eta_{n}\right) P_{C}\left(z_{n}-\delta_{n} B z_{n}\right)-p \|^{2} \\
= & \alpha_{n}\left\|f\left(T_{\mu_{n}} W_{n} y_{n}\right)-p\right\|^{2}+\beta_{n}\left\|x_{n}-p\right\|^{2}+\gamma_{n} \| \eta_{n}\left[P_{C}\left(z_{n}-\zeta_{n} A z_{n}\right)\right. \\
& \left.-P_{C}\left(p-\zeta_{n} A p\right)\right]+\left(1-\eta_{n}\right)\left[P_{C}\left(z_{n}-\delta_{n} B z_{n}\right)-P_{C}\left(p-\delta_{n} B p\right)\right] \|^{2} .
\end{aligned}
$$

From (26), we have

$$
\begin{aligned}
\| x_{n+1} & -p \|^{2} \\
\leq & \alpha_{n}\left\|f\left(T_{\mu_{n}} W_{n} y_{n}\right)-p\right\|^{2}+\beta_{n}\left\|x_{n}-p\right\|^{2} \\
& +\gamma_{n}\left[\eta_{n}\left\|\left(z_{n}-p\right)-\zeta_{n}\left(A z_{n}-A p\right)\right\|^{2}+\left(1-\eta_{n}\right)\left\|z_{n}-p\right\|^{2}\right] \\
= & \alpha_{n}\left\|f\left(T_{\mu_{n}} W_{n} y_{n}\right)-p\right\|^{2}+\beta_{n}\left\|x_{n}-p\right\|^{2} \\
& +\gamma_{n}\left(1-\eta_{n}\right)\left\|z_{n}-p\right\|^{2}+\gamma_{n} \eta_{n}\left[\left\|z_{n}-p\right\|^{2}+\zeta_{n}^{2}\left\|A z_{n}-A p\right\|^{2}\right. \\
& \left.-2 \zeta_{n}\left\langle A z_{n}-A p, z_{n}-p\right\rangle\right] \\
\leq & \alpha_{n}\left\|f\left(T_{\mu_{n}} W_{n} y_{n}\right)-p\right\|^{2}+\beta_{n}\left\|x_{n}-p\right\|^{2} \\
& +\gamma_{n}\left(1-\eta_{n}\right)\left\|z_{n}-p\right\|^{2}+\gamma_{n} \eta_{n}\left[\left\|z_{n}-p\right\|^{2}+\zeta_{n}^{2}\left\|A z_{n}-A p\right\|^{2}\right. \\
& \left.-2 \zeta_{n} \beta\left\|A z_{n}-A p, z_{n}-p\right\|^{2}\right] \\
= & \alpha_{n}\left\|f\left(T_{\mu_{n}} W_{n} y_{n}\right)-p\right\|^{2}+\beta_{n}\left\|x_{n}-p\right\|^{2} \\
& +\gamma_{n}\left\|z_{n}-p\right\|^{2}+\zeta_{n}\left(\zeta_{n}-2 \beta\right)\left\|A z_{n}-A p\right\|^{2} \\
\leq & \left\|x_{n}-p\right\|^{2}+\alpha_{n}\left[\left\|f\left(T_{\mu_{n}} W_{n} y_{n}\right)-p\right\|^{2}-\left\|x_{n}-p\right\|^{2}\right] \\
& +\zeta_{n}\left(\zeta_{n}-2 \beta\right)\left\|A z_{n}-A p\right\|^{2},
\end{aligned}
$$


which implies that

$$
\begin{aligned}
-\zeta_{n}\left(\zeta_{n}-2 \beta\right)\left\|A z_{n}-A p\right\|^{2} \\
\leq
\end{aligned}
$$

Therefore, from step 4 and condition $B_{1}$, we obtain

$$
\lim _{n \rightarrow \infty}\left\|A z_{n}-A p\right\|=0 .
$$

On the other hand from (26), we have

$$
\begin{aligned}
\| x_{n+1}- & p \|^{2} \\
\leq & \alpha_{n}\left\|f\left(T_{\mu_{n}} W_{n} y_{n}\right)-p\right\|^{2}+\beta_{n}\left\|x_{n}-p\right\|^{2}+\gamma_{n}\left[\eta_{n}\left\|z_{n}-p\right\|^{2}\right. \\
& \left.+\left(1-\eta_{n}\right)\left\|\left(z_{n}-p\right)-\delta_{n}\left(B z_{n}-B p\right)\right\|^{2}\right] \\
= & \alpha_{n}\left\|f\left(T_{\mu_{n}} W_{n} y_{n}\right)-p\right\|^{2}+\beta_{n}\left\|x_{n}-p\right\|^{2}+\gamma_{n}\left[\eta_{n}\left\|z_{n}-p\right\|^{2}\right. \\
& \left.+\left(1-\eta_{n}\right)\left(\left\|z_{n}-p\right\|^{2}-2 \delta_{n}\left\langle B z_{n}-B p, z_{n}-p\right\rangle+\delta_{n}^{2}\left\|B z_{n}-B p\right\|^{2}\right)\right] \\
\leq & \alpha_{n}\left\|f\left(T_{\mu_{n}} W_{n} y_{n}\right)-p\right\|^{2}+\beta_{n}\left\|x_{n}-p\right\|^{2}+\gamma_{n}\left[\eta_{n}\left\|z_{n}-p\right\|^{2}\right. \\
& \left.+\left(1-\eta_{n}\right)\left(\left\|z_{n}-p\right\|^{2}-2 \delta_{n} \gamma\left\|B z_{n}-B p\right\|^{2}+\delta_{n}^{2}\left\|B z_{n}-B p\right\|^{2}\right)\right] \\
= & \alpha_{n}\left\|f\left(T_{\mu_{n}} W_{n} y_{n}\right)-p\right\|^{2}+\beta_{n}\left\|x_{n}-p\right\|^{2}+\gamma_{n}\left\|z_{n}-p\right\|^{2} \\
& +\delta_{n}\left(\delta_{n}-2 \gamma\right) \gamma_{n}\left(1-\eta_{n}\right)\left\|B z_{n}-B p\right\|^{2} \\
\leq & \left\|x_{n}-p\right\|^{2}+\alpha_{n}\left[\left\|f\left(T_{\mu_{n}} W_{n} y_{n}\right)-p\right\|^{2}-\left\|x_{n}-p\right\|^{2}\right] \\
& +\delta_{n}\left(\delta_{n}-2 \gamma\right)\left\|B z_{n}-B p\right\|^{2}
\end{aligned}
$$

which implies that

$$
\begin{aligned}
-\delta_{n}\left(\delta_{n}-2 \gamma\right)\left\|B z_{n}-B p\right\|^{2} \\
\leq
\end{aligned}
$$

Therefore, from step 4 and condition $B_{1}$, we obtain

$$
\lim _{n \rightarrow \infty}\left\|B z_{n}-B p\right\|=0 .
$$

From (6) and (12), we have

$$
\begin{aligned}
&\left\|v_{n}-p\right\|^{2} \\
&=\left\|P_{C}\left(z_{n}-\zeta_{n} A z_{n}\right)-P_{C}\left(p-\zeta_{n} A p\right)\right\|^{2} \\
& \leq\left\langle\left(z_{n}-\zeta_{n} A z_{n}\right)-\left(p-\zeta_{n} A p\right), v_{n}-p\right\rangle \\
&= \frac{1}{2}\left[\left\|\left(z_{n}-\zeta_{n} A z_{n}\right)-\left(p-\zeta_{n} A p\right)\right\|^{2}+\left\|v_{n}-p\right\|^{2}\right. \\
&\left.-\left\|\left(z_{n}-\zeta_{n} A z_{n}\right)-\left(p-\zeta_{n} A p\right)-\left(v_{n}-p\right)\right\|^{2}\right] \\
&= \frac{1}{2}\left[\left\|z_{n}-p\right\|^{2}+\left\|v_{n}-p\right\|^{2}-\left\|z_{n}-v_{n}\right\|^{2}\right. \\
&\left.+2 \zeta_{n}\left\langle z_{n}-v_{n}, A z_{n}-A p\right\rangle-\zeta_{n}^{2}\left\|A z_{n}-A p\right\|^{2}\right] .
\end{aligned}
$$


So we obtain

$$
\begin{aligned}
\left\|v_{n}-p\right\|^{2} \leq & \left\|z_{n}-p\right\|^{2}-\left\|z_{n}-v_{n}\right\|^{2} \\
& +2 \zeta_{n}\left\langle z_{n}-v_{n}, A z_{n}-A p\right\rangle-\zeta_{n}^{2}\left\|A z_{n}-A p\right\|^{2} .
\end{aligned}
$$

By using the same method as (29), we have

$$
\begin{aligned}
\left\|w_{n}-p\right\|^{2} \leq & \left\|z_{n}-p\right\|^{2}-\left\|z_{n}-w_{n}\right\|^{2} \\
& +2 \delta_{n}\left\langle z_{n}-w_{n}, B z_{n}-B p\right\rangle-\delta_{n}^{2}\left\|B z_{n}-B p\right\|^{2}
\end{aligned}
$$

From (29), (30) and definition of $y_{n}$, we have,

$$
\begin{aligned}
&\left\|y_{n}-p\right\|^{2} \\
&= \| \eta_{n}\left[P_{C}\left(z_{n}-\zeta_{n} A z_{n}\right)-p\right] \\
&+\left(1-\eta_{n}\right)\left[P_{C}\left(z_{n}-\delta_{n} B z_{n}\right)-p\right] \|^{2} \\
&=\left\|\eta_{n}\left(v_{n}-p\right)+\left(1-\eta_{n}\right)\left(w_{n}-p\right)\right\|^{2} \\
& \leq \eta_{n}\left\|v_{n}-p\right\|^{2}+\left(1-\eta_{n}\right)\left\|w_{n}-p\right\|^{2} \\
& \leq \eta_{n}\left[\left\|z_{n}-p\right\|^{2}-\left\|z_{n}-v_{n}\right\|^{2}+2 \zeta_{n}\left\langle z_{n}-v_{n}, A z_{n}-A p\right\rangle\right. \\
&\left.-\zeta_{n}^{2}\left\|A z_{n}-A p\right\|^{2}\right]+\left(1-\eta_{n}\right)\left[\left\|z_{n}-p\right\|^{2}-\left\|z_{n}-w_{n}\right\|^{2}\right. \\
&\left.+2 \delta_{n}\left\langle z_{n}-w_{n}, B z_{n}-B p\right\rangle-\delta_{n}^{2}\left\|B z_{n}-B p\right\|^{2}\right] \\
& \leq\left\|z_{n}-p\right\|^{2}+\eta_{n}\left[-\left\|z_{n}-v_{n}\right\|^{2}+2 \zeta_{n}\left\|z_{n}-v_{n}\right\|\left\|A z_{n}-A p\right\|\right. \\
&\left.-\zeta_{n}^{2}\left\|A z_{n}-A p\right\|^{2}\right]+\left(1-\eta_{n}\right)\left[-\left\|z_{n}-w_{n}\right\|^{2}\right. \\
&\left.+2 \delta_{n}\left\|z_{n}-w_{n}\right\|\left\|B z_{n}-B p\right\|-\delta_{n}^{2}\left\|B z_{n}-B p\right\|^{2}\right]
\end{aligned}
$$

By (31), we have

$$
\begin{aligned}
\| x_{n+1} & -p \|^{2} \\
\leq & \alpha_{n}\left\|f\left(T_{\mu_{n}} W_{n} y_{n}\right)-p\right\|^{2}+\beta_{n}\left\|x_{n}-p\right\|^{2}+\gamma_{n}\left\|T_{\mu_{n}} W_{n} \mathcal{J}_{n}^{M} y_{n}-p\right\|^{2} \\
\leq & \alpha_{n}\left\|f\left(T_{\mu_{n}} W_{n} y_{n}\right)-p\right\|^{2}+\beta_{n}\left\|x_{n}-p\right\|^{2}+\gamma_{n}\left\|z_{n}-p\right\|^{2} \\
& +\gamma_{n} \eta_{n}\left[-\left\|z_{n}-v_{n}\right\|^{2}+2 \zeta_{n}\left\|z_{n}-v_{n}\right\|\left\|A z_{n}-A p\right\|\right. \\
& \left.-\zeta_{n}^{2}\left\|A z_{n}-A p\right\|^{2}\right]+\gamma_{n}\left(1-\eta_{n}\right)\left[-\left\|z_{n}-w_{n}\right\|^{2}\right. \\
& \left.+2 \delta_{n}\|\| z_{n}-w_{n}\|\| B z_{n}-B p\left\|-\delta_{n}^{2}\right\| B z_{n}-B p \|^{2}\right] \\
\leq & \alpha_{n}\left\|f\left(T_{\mu_{n}} W_{n} y_{n}\right)-p\right\|^{2}+\beta_{n}\left\|x_{n}-p\right\|^{2}+\gamma_{n}\left\|x_{n}-p\right\|^{2} \\
& -\gamma_{n} \eta_{n}\left\|z_{n}-v_{n}\right\|^{2}+\gamma_{n} \eta_{n}\left[2 \zeta_{n}\left\|z_{n}-v_{n}\right\|\left\|A z_{n}-A p\right\|^{2}\right. \\
& \left.-\zeta_{n}^{2}\left\|A z_{n}-A p\right\|^{2}\right]-\gamma_{n}\left(1-\eta_{n}\right)\left\|z_{n}-w_{n}\right\|^{2} \\
& +\gamma_{n}\left(1-\eta_{n}\right)\left[2 \delta_{n}\|\| z_{n}-w_{n}\|\| B z_{n}-B p\left\|-\delta_{n}^{2}\right\| B z_{n}-B p \|^{2}\right] \\
= & \left\|x_{n}-p\right\|^{2}+\alpha_{n}\left[\left\|f\left(T_{\mu_{n}} W_{n} y_{n}\right)-p\right\|^{2}-\left\|x_{n}-p\right\|^{2}\right] \\
& -\gamma_{n} \eta_{n}\left\|z_{n}-v_{n}\right\|^{2}+\gamma_{n} \eta_{n}\left[2 \zeta_{n}\left\|z_{n}-v_{n}\right\|\left\|A z_{n}-A p\right\|\right. \\
& \left.-\zeta_{n}^{2}\left\|A z_{n}-A p\right\|^{2}\right]-\gamma_{n}\left(1-\eta_{n}\right)\left\|z_{n}-w_{n}\right\|^{2} \\
& +\gamma_{n}\left(1-\eta_{n}\right)\left[2 \delta_{n}\|\| z_{n}-w_{n}\|\| B z_{n}-B p\left\|-\delta_{n}^{2}\right\| B z_{n}-B p \|^{2}\right]
\end{aligned}
$$


which implies that

$$
\begin{aligned}
\gamma_{n} \eta_{n} & \left\|z_{n}-v_{n}\right\|^{2} \\
\leq & {\left[\left\|x_{n}-p\right\|+\left\|x_{n+1}-p\right\|\right]\left\|x_{n+1}-x_{n}\right\| } \\
& +\alpha_{n}\left[\left\|f\left(T_{\mu_{n}} W_{n} y_{n}\right)-p\right\|^{2}-\left\|x_{n}-p\right\|^{2}\right] \\
& +\gamma_{n} \eta_{n}\left[2 \zeta_{n}\left\|z_{n}-v_{n}\right\|\left\|A z_{n}-A p\right\|-\zeta_{n}^{2}\left\|A z_{n}-A p\right\|^{2}\right] \\
& +\gamma_{n}\left(1-\eta_{n}\right)\left[2 \delta_{n}\|\| z_{n}-w_{n}\|\| B z_{n}-B p\left\|-\delta_{n}^{2}\right\| B z_{n}-B p \|^{2}\right],
\end{aligned}
$$

and

$$
\begin{aligned}
\gamma_{n}(1 & \left.-\eta_{n}\right)\left\|z_{n}-w_{n}\right\|^{2} \\
\leq & {\left[\left\|x_{n}-p\right\|+\left\|x_{n+1}-p\right\|\right]\left\|x_{n+1}-x_{n}\right\| } \\
& +\gamma_{n} \eta_{n}\left[2 \zeta_{n}\left\|z_{n}-v_{n}\right\|\left\|A z_{n}-A p\right\|-\zeta_{n}^{2}\left\|A z_{n}-A p\right\|^{2}\right] \\
& +\gamma_{n}\left(1-\eta_{n}\right)\left[2 \delta_{n}\|\| z_{n}-w_{n}\|\| B z_{n}-B p\left\|-\delta_{n}^{2}\right\| B z_{n}-B p \|^{2}\right] .
\end{aligned}
$$

Therefore, from $0<\lim _{\inf } f_{n \rightarrow \infty} \gamma_{n} \leq \lim \sup _{n \rightarrow \infty} \gamma_{n}<1$, condition $B_{1}$, step 4, (27) and (28) we get

$$
\lim _{n \rightarrow \infty}\left\|z_{n}-v_{n}\right\|=0 \text { and } \lim _{n \rightarrow \infty}\left\|z_{n}-w_{n}\right\|=0 .
$$

Let $U: H \rightarrow 2^{H}$ be a set-valued mapping is defined by

$$
U x= \begin{cases}A x+N_{C} x, & x \in C, \\ \emptyset, & x \notin C,\end{cases}
$$

where $N_{C} x$ is the normal cone to $C$ at $x \in C$. Since $A$ is monotone. Thus $U$ is maximal monotone see [1]. Let $(x, y) \in G(U)$, hence $y-A x \in N_{C} x$ and since $v_{n}=P_{C}\left(z_{n}\right.$ $\left.\zeta_{n} A z_{n}\right)$ therefore, $\left\langle x-v_{n}, y-A x\right\rangle \geq 0$. On the other hand from (7), we have

$$
\left\langle x-v_{n}, v_{n}-\left(z_{n}-\zeta_{n} A z_{n}\right)\right\rangle \geq 0,
$$

i.e.,

$$
\left\langle x-v_{n}, \frac{v_{n}-z_{n}}{\zeta_{n}}+A z_{n}\right\rangle \geq 0
$$

Therefore, we have

$$
\begin{aligned}
\langle x & \left.-v_{n_{i}}, y\right\rangle \\
& \geq\left\langle x-v_{n_{i}}, A x\right\rangle \\
& \geq\left\langle x-v_{n_{i}}, A x\right\rangle-\left\langle x-v_{n_{i}}, \frac{v_{n_{i}}-z_{n_{i}}}{\zeta_{n_{i}}}+A z_{n_{i}}\right\rangle \\
& =\left\langle x-v_{n_{i}}, A x-\frac{v_{n_{i}}-z_{n_{i}}}{\zeta_{n_{i}}}-A z_{n_{i}}\right\rangle \\
& =\left\langle x-v_{n_{i}}, A x-A v_{n_{i}}\right\rangle+\left\langle x-v_{n_{i}}, A v_{n_{i}}-A z_{n_{i}}\right\rangle-\left\langle x-v_{n_{i}}, \frac{v_{n_{i}}-z_{n_{i}}}{\zeta_{n_{i}}}\right\rangle \\
& \geq\left\langle x-v_{n_{i}}, A v_{n_{i}}-A z_{n_{i}}\right\rangle-\left\langle x-v_{n_{i}}, \frac{v_{n_{i}}-z_{n_{i}}}{\zeta_{n_{i}}}\right\rangle \\
& \geq\left\langle x-v_{n_{i}}, A v_{n_{i}}-A z_{n_{i}}\right\rangle-\left\|x-v_{n_{i}}\right\|\left\|\frac{v_{n_{i}}-z_{n_{i}}}{\zeta_{n_{i}}}\right\| .
\end{aligned}
$$


From (32), we get $\lim _{i \rightarrow \infty}\left\|v_{n_{i}}-z_{n_{i}}\right\|=0$. Noting that $x_{n_{i}} \rightarrow z$ and $A$ is $\frac{1}{\beta}$-lipschitzian, we obtain

$$
\langle x-z, y\rangle \geq 0 .
$$

Since $U$ is maximal monotone, we have $z \in U^{-1} 0$, and hence $z \in \operatorname{VI}(C, A)$. Let $V: H \rightarrow$ $2^{H}$ be a set-valued mapping is defined by

$$
V x= \begin{cases}B x+N_{C} x, & x \in C, \\ \emptyset, & x \notin C,\end{cases}
$$

where $N_{C} x$ is the normal cone to $C$ at $x \in C$. Since $B$ is monotone. Thus $U$ is maximal monotone see [1]. Repeating the same argument as above, we can derive $z \in \mathrm{VI}$ $(C, B)$. Therefore, $z \in \mathcal{F}$.

Step 9. There exists a unique $x^{*} \in C$ such that

$$
\limsup _{n \rightarrow \infty}\left\langle f\left(x^{*}\right)-x^{*}, x_{n}-x^{*}\right\rangle \leq 0 .
$$

Proof of Step 9. Note that $f$ is a contraction mapping with coefficient $\alpha \in(0,1)$. Then $\left\|P_{\mathcal{F}} f(x)-P_{\mathcal{F}} f(y)\right\| \leq\|f(x)-f(y)\| \leq \alpha\|x-y\|$ for all $x, y \in H$. Therefore $P_{\mathcal{F}}$ is a contraction of $H$ into itself, which implies that there exists a unique element $x^{*} \in H$ such that $x^{*}=P_{\mathcal{F}} f\left(x^{*}\right)$. at the same time, we note that $x^{*} \in C$. Using Lemma 2.3, we have

$$
\left\langle f\left(x^{*}\right)-x^{*}, x^{*}-z\right\rangle \geq 0, \quad \forall z \in \mathcal{F} .
$$

We can choose a subsequence $\left\{x_{n_{k}}\right\}$ of $\left\{x_{n}\right\}$ such that

$$
\limsup _{n \rightarrow \infty}\left\langle f\left(x^{*}\right)-x^{*}, x_{n}-x^{*}\right\rangle=\lim _{k \rightarrow \infty}\left\langle f\left(x^{*}\right)-x^{*}, x_{n_{k}}-x^{*}\right\rangle .
$$

Since $\left\{x_{n_{k}}\right\}$ is bounded, therefore, $\left\{x_{n_{k}}\right\}$ has subsequence $\left\{x_{n_{k_{j}}}\right\}$ such that $x_{n_{k_{j}}} \rightarrow z^{*}$. With no loss of generality, we may assume that $x_{n_{k}} \rightarrow z^{*}$. Applying Step 8 and (34), we have

$$
\limsup _{n \rightarrow \infty}\left\langle f\left(x^{*}\right)-x^{*}, x_{n}-x^{*}\right\rangle=\left\langle f\left(x^{*}\right)-x^{*}, z^{*}-x^{*}\right\rangle \leq 0 .
$$

Step 10, The sequences $\left\{x_{n}\right\}$ converges strongly to $x^{*}$, which is obtained in Steep 9. Proof of Step 10. We have

$$
\begin{aligned}
&\left\|x_{n+1}-x^{*}\right\|^{2} \\
&=\left\|\alpha_{n}\left(f\left(T_{\mu_{n}} W_{n} y_{n}\right)-x^{*}\right)+\beta_{n}\left(x_{n}-x^{*}\right)+\gamma_{n}\left(T_{\mu_{n}} W_{n} y_{n}-x^{*}\right)\right\|^{2} \\
& \leq\left\|\beta_{n}\left(x_{n}-x^{*}\right)+\gamma_{n}\left(T_{\mu_{n}} W_{n} y_{n}-x^{*}\right)\right\|^{2}+2 \alpha_{n}\left\langle f\left(T_{\mu_{n}} W_{n} y_{n}\right)-x^{*}, x_{n+1}-x^{*}\right\rangle \\
& \leq {\left[\beta_{n}\left\|x_{n}-x^{*}\right\|+\gamma_{n}\left\|T_{\mu_{n}} W_{n} y_{n}-x^{*}\right\|\right]^{2} } \\
&+2 \alpha_{n}\left\langle f\left(T_{\mu_{n}} W_{n} y_{n}\right)-f\left(x^{*}\right), x_{n+1}-x^{*}\right\rangle+2 \alpha_{n}\left\langle f\left(x^{*}\right)-x^{*}, x_{n+1}-x^{*}\right\rangle \\
& \leq {\left[\beta_{n}\left\|x_{n}-x^{*}\right\|+\gamma_{n}\left\|y_{n}-x^{*}\right\|\right]^{2} } \\
&+2 \alpha_{n} \alpha\left\|y_{n}-x^{*}\right\|\left\|x_{n+1}-x^{*}\right\|+2 \alpha_{n}\left\langle f\left(x^{*}\right)-x^{*}, x_{n+1}-x^{*}\right\rangle \\
& \leq {\left[\beta_{n}\left\|x_{n}-x^{*}\right\|+\gamma_{n}\left\|x_{n}-x^{*}\right\|\right]^{2} } \\
&+2 \alpha_{n} \alpha\left\|x_{n}-x^{*}\right\|\left\|x_{n+1}-x^{*}\right\|+2 \alpha_{n}\left\langle f\left(x^{*}\right)-x^{*}, x_{n+1}-x^{*}\right\rangle \\
&=\left(1-\alpha_{n}\right)^{2}\left\|x_{n}-x^{*}\right\|^{2}+\alpha_{n} \alpha\left[\left\|x_{n}-x^{*}\right\|^{2}+\left\|x_{n+1}-x^{*}\right\|^{2}\right] \\
&+2 \alpha_{n}\left\langle f\left(x^{*}\right)-x^{*}, x_{n+1}-x^{*}\right\rangle \\
&
\end{aligned}
$$


Which implies that

$$
\begin{aligned}
& \left\|x_{n+1}-x^{*}\right\|^{2} \\
& \quad \leq \frac{\left(1-\alpha_{n}\right)^{2}+\alpha_{n} \alpha}{1-\alpha_{n} \alpha}\left\|x_{n}-x^{*}\right\|^{2}+\frac{2 \alpha_{n}}{1-\alpha_{n} \alpha}\left\langle f\left(x^{*}\right)-x^{*}, x_{n+1}-x^{*}\right\rangle \\
& \quad=\left(1-\alpha_{n} \frac{2-2 \alpha}{1-\alpha_{n} \alpha}\right)\left\|x_{n}-x^{*}\right\|^{2}+\alpha_{n} \tau_{n}
\end{aligned}
$$

where

$$
\tau_{n}=\frac{\alpha_{n}}{1-\alpha_{n} \alpha}\left\|x_{n}-x^{*}\right\|^{2}+\frac{2}{1-\alpha_{n} \alpha}\left\langle f\left(x^{*}\right)-x^{*}, x_{n+1}-x^{*}\right\rangle,
$$

By Step 9, and condition $\left(B_{1}\right)$, we get $\lim \sup _{n \rightarrow \infty} \tau_{n} \leq 0$. Now applying Lemma 2.8 to (35), we conclude that $x_{n} \rightarrow x^{*}$. Consequently, from $\left\|J_{r_{k}, n}^{F_{k}} x_{n}-x^{*}\right\| \leq\left\|x_{n}-x^{*}\right\|$, we have $J_{r_{k}, n}^{F_{k}} x_{n} \rightarrow x^{*}$, for all $k \in\{1,2, \ldots, M\}$.

Corollary 3.2. (see Yao et al. [8]) Let $C$ be a nonempty closed convex subset of a real Hilbert space $H, F$ a bi-functions from $C \times C$ into $\mathbb{R}$ which satisfy $\left(A_{1}\right)-\left(A_{4}\right)$ and $\left\{T_{i}\right\}_{i=1}^{\infty}$ an infinite family of nonexpansive mapping of $C$ into $C$ such that $\cap_{i=1}^{\infty} \operatorname{Fix}\left(T_{i}\right) \cap \operatorname{EP}(F) \neq \emptyset$. Let $\left\{\alpha_{n}\right\},\left\{\beta_{n}\right\}$ and $\left\{\gamma_{n}\right\}$ are three sequences in $(0,1)$ such that $\alpha_{n}+\beta_{n}+\gamma_{n}=1$ and $\left\{r_{n}\right\} \subset(0, \infty)$. Suppose the following conditions are satisfied:

$\left(\mathrm{B}_{1}\right) \lim _{n \rightarrow \infty} \alpha_{n}=0$ and $\sum_{n=1}^{\infty} \alpha_{n}=\infty$,

$\left(\mathrm{B}_{2}\right) 0<\lim \inf _{n \rightarrow \infty} \beta_{n} \leq \lim \sup _{n \rightarrow \infty} \beta_{n}<1$,

$\left(\mathrm{B}_{3}\right) \lim \inf _{n \rightarrow \infty} r_{n}>0$ and $\lim _{n \rightarrow \infty}\left(r_{n+1}-r_{n}\right)=0$.

Let $f$ be a contraction of $C$ into itself with coefficient $\alpha \in(0,1)$ and given $x_{1} \in C$ arbitrarily. Then the sequence $\left\{x_{n}\right\}$ generated by

$$
x_{n+1}=\alpha_{n} f\left(x_{n}\right)+\beta_{n} x_{n}+\gamma_{n} W_{n} J_{r_{n}}^{F} x_{n}, n \geq 1 .
$$

converge strongly to $x^{*} \in \cap_{i=1}^{\infty} \operatorname{Fix}\left(T_{i}\right) \cap \operatorname{EP}(F)$, where $x^{*} \in P_{\cap_{i=1}^{\infty} \operatorname{Fix}\left(T_{i}\right) \cap \operatorname{EP}(F)} f\left(x^{*}\right)$.

Proof. Take $A=B=0, \phi=\{I\}, F_{1}=F$ and $F_{k}=0$ for $k \in\{2, \ldots, M\}$ in Theorem 3.1, then we have $T_{\mu_{n}}=I$ and $y_{n}=z_{n}=J_{r_{m}}^{k} x_{n}$. So from Theorem 3.1 the sequence $\left\{x_{n}\right\}$ converges strongly to $x^{*} \in \cap_{i=1}^{\infty} \operatorname{Fix}\left(T_{i}\right) \cap \operatorname{EP}(F)$, where $x^{*} \in P_{\bigcap_{i=1}^{\infty} \operatorname{Fix}\left(T_{i}\right) \cap \operatorname{EP}(F)} f\left(x^{*}\right)$.

Corollary 3.3. Let $C$ be a nonempty closed convex subset of a real Hilbert space $H$, $\mathcal{J}=\left\{F_{k}: k=1,2, \ldots, M\right\}$ be a finite family of bi-functions from $C \times C$ into $\mathbb{R}$ which satisfy $\left(A_{1}\right)-\left(A_{4}\right), T$ a nonexpansive mappings on $C$ such that $\operatorname{Fix}(T) \cap \operatorname{EP}(\mathcal{J}) \neq \emptyset$. Let $\left\{\alpha_{n}\right\},\left\{\beta_{n}\right\}$ and $\left\{\gamma_{n}\right\}$ are three sequences in $(0,1)$ such that $\alpha_{n}+\beta_{n}+\gamma_{n}=1$ and $\left\{r_{k, n}\right\}_{k=1}^{M}$ be sequences in $(0, \infty)$. Suppose the following conditions are satisfied:

$\left(\mathrm{B}_{1}\right) \lim _{n \rightarrow \infty} \alpha_{n}=0$ and $\sum_{n=1}^{\infty} \alpha_{n}=\infty$,

$\left(\mathrm{B}_{2}\right) 0<\lim \inf _{n \rightarrow \infty} \beta_{n} \leq \lim \sup _{n \rightarrow \infty} \beta_{n}<1$,

$\left(\mathrm{B}_{3}\right) \lim \inf _{n \rightarrow \infty} r_{k, n}>0$ and $\lim _{n \rightarrow \infty}\left(r_{k, n+1}-r_{k, n}\right)=0$ for $k \in\{1,2, \ldots, M\}$.

Let $f$ be a contraction of $H$ into itself and given $x_{1} \in H$ arbitrarily. If the sequences $\left\{x_{n}\right\}$ generated iteratively by

$$
x_{n+1}=\alpha_{n} f\left(x_{n}\right)+\beta_{n} x_{n}+\gamma_{n} \frac{1}{n} \sum_{k=0}^{\infty}\left(\frac{n-1}{n}\right)^{k} T^{k} J_{r_{M, n}}^{F_{M}} \ldots J_{r_{2 n}}^{F_{2}} J_{r_{1 n}}^{F_{1}}, x_{n}, \quad n \geq 1 .
$$


Then, sequences $\left\{x_{n}\right\}$ and $\left\{J_{r_{k}, n}^{F_{k}} x_{n}\right\}_{k=1}^{M}$ converge strongly to $x^{*} \in \operatorname{Fix}(T) \cap \operatorname{EP}(\mathcal{J})$, where $x^{*}=P_{\operatorname{Fix}(T) \cap \operatorname{EP}(\mathcal{J})} f\left(x^{*}\right)$.

Proof. Let $S=\{0,1, \ldots\}, \phi=\left\{T^{i}: i \in S\right\}$ and $T^{0}=I$. For $f=\left(x_{0}, x_{1}, \ldots\right) \in B(S)$, define

$$
\mu_{n}(f)=\frac{1}{n} \sum_{k=0}^{\infty}\left(\frac{n-1}{n}\right)^{k} x_{k}, \quad \forall n \in \mathbb{N} .
$$

Then $\left\{\mu_{n}\right\}$ is a regular sequence of means on $B(S)$ such that $\lim _{n \rightarrow \infty}\left\|\mu_{n+}-\mu_{n}\right\|=0$; for more details, see [34]. Next for each $x \in H$ and $n \in \mathbb{N}$, we have

$$
T_{\mu_{n}} x=\frac{1}{n} \sum_{k=0}^{\infty}\left(\frac{n-1}{n}\right)^{k} T^{k} x .
$$

Take $A=B=0, T_{i}=I$ for all $i \in \mathbb{N}$ in Theorem 3.1 then we have $y_{n}=z_{n}$ and $W_{n}=$ $I$ for all $n \in \mathbb{N}$. Therefore, it follows from Theorem 3.1 that the sequences $\left\{x_{n}\right\}$ and $\left\{J_{r_{k}, n}^{F_{k}} x_{n}\right\}_{k=1}^{M}$ converge strongly, as $n \rightarrow \infty$ to a point $x^{*} \in \operatorname{Fix}(T) \cap \operatorname{EP}(\mathcal{J})$, where $x^{*}=P_{\operatorname{Fix}(T) \cap E P(\mathcal{J})} f\left(x^{*}\right)$.

Remark 3.4. Theorem 3.1 improve [8, Theorem 1.2] in the following aspects.

(a) Our iterative process (11) is more general than Yao et al. process (14) because it can be applied to solving the problem of finding a common element of the set of solutions of systems of equilibrium problems and systems of variational inequalities.

(b) Our iterative process (11) is very diffident from Yao et al. process (14) because there are left amenable semigroup of nonexpansive mappings.

(c) Our method of proof is very different from the on in Yao et al. [8] for example we use Corollary 1.1 and Theorem 1.2 of Bruck [33] fore the proof of Theorem 3.1.

\section{Acknowledgements}

The authors are extremely grateful to the referees for useful suggestions that improved the contents of the paper.

\section{Competing interests}

The authors declare that they have no competing interests.

Received: 31 December 2011 Accepted: 16 June 2012 Published: 16 June 2012

\section{References}

1. Rockafellar, RT: On the maximality of sums of nonlinear monotone operators. Trans Am Math Soc. 149, 75-88 (1970). doi:10.1090/S0002-9947-1970-0282272-5

2. Blum, E, Oettli, W: From optimization and variational inequalities to equilibrium problems. Math Student. 63, 123-145 (1994)

3. Combettes, PL, Hirstoaga, SA: Equilibrium programming in Hilbert spaces. J Nonlinear Convex Anal. 6, 117-136 (2005)

4. Flåm, SD, Antipin, AS: Equilibrium programming using proximal-like algorithms. Math Programm. 78, 29-41 (1997)

5. Takahashi, S, Takahashi, W: Viscosity approximation methods for equilibrium problems and fixed point problems in Hilbert spaces. J Math Anal Appl. 331, 506-515 (2007). doi:10.1016/j.jmaa.2006.08.036

6. Bauschke, HH, Borwein, JM: On projection algorithms for solving convex feasibility problems. SIAM Rev. 38, 367-426 (1996). doi:10.1137/S0036144593251710

7. Combettes, PL: Quasi-fejérian analysis of some optimization algorithms. Inherently Parallel Algorithms in Feasibility and Optimization and Their Applications (Haifa, 2000). In Studies in Computational Mathematics, vol. 8, pp. 115-152.NorthHolland, Amsterdam (2001)

8. Yao, Y, Liou, YC, Yao, JC: Convergence theorem for equilibrium problems and fixed point problems of infinite family of nonexpansive mappings. Fixed Point Theory Appl 12 (2007). Article ID 64363

9. Jitpeera, T, Katchang, P, Kumam, P: A Viscosity of Ceaàro Mean Approximation Method for a Mixed Equilibrium. Variational Inequality, and Fixed Point Problems 2011, 24 (2011). Article ID 945051, 838.85. MR 2680251

10. Yao, Y, Chen, R, Liou, YC: A unified implicit algorithm for solving the triple-hierarchical constrained optimization problem. Math Comput Model. 55, 1506-1515 (2012). doi:10.1016/j.mcm.2011.10.041

11. Yao, Y, Chen, R, Xu, HK: Schemes for finding minimum-norm solutions of variational inequalities. Nonlinear Anal. 72 3447-3456 (2010). doi:10.1016/j.na.2009.12.029 
12. Yao, Y, Cho, YJ, Chen, R: An iterative algorithm for solving fixed point problems, variational inequality problems and mixed equilibrium problems. Nonlinear Anal. 71, 3363-3373 (2009). doi:10.1016/j.na.2009.01.236

13. Yao, Y, Cho, YJ, Liou, YC: Hierarchical convergence of an implicit double-net algorithm for nonexpansive semigroups and variational inequalities. Fixed Point Theory Appl. 2011 (2011)

14. Yao, Y, Liou, YC, Kang, SM: Two-step projection methods for a system of variational inequality problems in Banach spaces. J Glob Optim

15. Yao, Y, Liou, YC, Marino, G: Strong convergence of two iterative algorithms for nonexpansive mappings in hilbert spaces. Fixed Point Theory Appl. (2009)

16. Lau, AT, Miyake, H, Takahashi, W: Approximation of fixed points for amenable semigroups of nonexpansive mappings in Banach spaces. Nonlinear Anal. 67, 1211-1225 (2007). doi:10.1016/j.na.2006.07.008

17. Kangtunyakarn, A: Iterative methods for finding common solution of generalized equilibrium problems and variational inequality problems and fixed point problems of a finite family of nonexpansive mappings. Fixed Point Theory Appl 2010, 29 (2010). Article ID 836714

18. Kim, KS: Convergence of a hybrid algorithm for a reversible semigroup of nonlinear operators in banach spaces. Nonlinear Anal. 73(3), 3413-3419 (2010)

19. Atsushiba, S, Takahashi, W: Approximating common fixed points of nonexpansive semigroups by the Mann iteration process. Ann Univ Mariae Curie Sklodowska. 51, 1-16 (1997)

20. Saeidi, S: Iterative algorithms for finding common solutions of variational inequalities and systems of equilibrium problems and fixed points of families and semigroups of nonexpansive mappings. Nonlinear Anal. 70, 4195-4208 (2009). doi:10.1016/j.na.2008.09.009

21. Piri, H: A general iterative method for finding common solutions of system of equilibrium problems, system of variational inequalities and fixed point problems. Math Comput Model. 55, 1622-1638 (2012). doi:10.1016/j. mcm.2011.10.069

22. Piri, $\mathrm{H}$ : Hybrid pseudoviscosity approximation schemes for systems of equilibrium problems and fixed point problems of infinitely and semigroup of nonexpansive mappings. Nonlinear Anal. 74, 6788-6804 (2011). doi:10.1016/j. na.2011.06.056

23. Piri, $\mathrm{H}$ : Strong convergence for a minimization problem on solutions of systems of equilibrium problems and common fixed points of an infinite family and semigroup of nonexpansive mappings. Comput Math Appl. 61, 2562-2577 (2011). doi:10.1016/j.camwa.2011.02.049

24. Piri, $\mathrm{H}$, Badali, AH: Strong convergence theorem for amenable semigroups of nonexpansive mappings and variational inequalities. Fixed Point Theory Appl. 2011 (2011)

25. Lau, AT, Shioji, N, Takahashi, W: Existence of nonexpansive retractions for amenable semigroups of nonexpansive mappings and nonlinear ergodic theorems in Banach spaces. J Funct Anal. 161(1), 62-75 (1999). doi:10.1006/ jfan.1998.3352

26. Agarwal, RP, Donal, O'Regan, Sahu, DR: Fixed point theory for Lipschitzian-type mappings with applications. In Topological Fixed Point Theory and Its Applications, vol. 6,Springer, New York (2009)

27. Jitpeera, T, Kumam, P: An extragradient type method for a system of equilibrium problems, variational inequality problems and fixed point of finitely many nonexpansive mappings. J Nonlinear Anal Optim. 1, 71-91 (2010)

28. Suzuki, T: Strong convergence of Krasnoselskii and Mann's type sequences for oneparameter nonexpansive semigroups without Bochner integrals. J Math Anal Appl. 305, 227-239 (2005). doi:10.1016/j.jmaa.2004.11.017

29. Shimoji, K, Takahashi, W: Strong convergence to common fixed points of infinite nonexpansive mappings and applications. Taiwan J Math. 5, 387-404 (2001)

30. Zhang, SS, Joseph Lee, HJ, Chan, CK: Algorithms of common solutions to quasi variational inclusion and fixed point problems. Appl Math Mech. 29, 571-581 (2008). doi:10.1007/s10483-008-0502-y

31. Xu, HK: Viscosity approximation methods for nonexpansive mappings. J Math Anal Appl. 298, 279-291 (2004). doi:10.1016/j.jmaa.2004.04.059

32. Osilike, MO, Igbokwe, DI: Weak and strong convergence theorems for fixed points of pseudocontractions and solutions of monotone type operator equations. Comput Math Appl. 40, 559-567 (2000). doi:10.1016/S0898-1221(00)00179-6

33. Bruck, RE: On the convex approximation property and the asymptotic behavior of nonlinear contractions in Banach spaces. Israel J Math. 38, 304-314 (1981). doi:10.1007/BF02762776

34. Takahashi, W: Nonlinear Functional Analysis. Yokohama Publishers, Yokohama (2000)

doi:10.1186/1687-1812-2012-99

Cite this article as: Piri: Approximating fixed points of amenable semigroup and infinite family of nonexpansive mappings and solving systems of variational inequalities and systems of equilibrium problems. Fixed Point Theory and Applications 2012 2012:99. 